\title{
Application of Reactivity Weight Factors to Reactor Transients
}

\author{
G. Lellouche, D. Diamond, \\ M. Levine
}

\author{
Brookhaven National Laboratory \\ Upton, New York 11973
}

\begin{abstract}
NOTICE
This raport was prapered as an account of work the United States thited States Govinment. Netther the United States nor the United Stutes Atomic Eneray
Commilalon, nor any of their employees, nor tholr contractors, subcontroter nor any of makes any wurzanty, exprestors; of thir employees, loinl linblity orranty, expreas or implied, or asumes any plat hility or. reapontibility for the eccuracy, com plotenow os urefulinen of any information, product or procesw. dieclosed or would not infinge privatoly owned presents thet fts use
\end{abstract}


Table of Content;

Introduction

page

I. Definitions and calculational Methods

Perturbation Theory

Equivalent Average Feedback

Westinghouse Method

Semi-Arbitrary Definition

Method of Eigenvalues

II. Weight Factors and Xenon Stability 16

III. Neight Factors and Inlet Coolant Temperature 25 Changes

IV. Partial Weight Factors 28

v. Doppler Weight Factors 31

Static Doppler Weiyht Factors * , 32

Predicting the Transient Doppler Weight Factor 36

Radial Calculations at the Axial Peak Fuel. 40 Temperature

Validation of Separability

Eigenvalue Comparison

Power Peak/Average Comparison

48

Doppler Weight Factor Comparison

vI. Conclusions 
Introduction

The change in reactivity produced by a small crosssection alteration is approximately proportional to the amount of the cross-section change and varies as a function of the position at which the change is made. This function of position, or weight, is usually strongly correlated in a positive sense with the power density in the reactor. Often it varies in the core approximately as the square of the power density. When we consider feedback in which the local power density produces temperature changes which affect the cross sections, it is necessary to take this correlation into account when performing averages. Otherwise, the amount of the feedback will be underestimated.

Mathematically, this can be expressed as follows. Iet $T$ and $W$ be the temperature and the weight as functions of position. And let a bar denote a spatial average. Then, the correlation described above means that

$$
\overline{(T-\bar{T})(W-\bar{W})}=\overline{T W}-\bar{T} \bar{W}>0
$$

and 
$\overline{T W} / \bar{T} \bar{W}=\mu$, a number greater than unity.

Since the reactivity effect is given by

$$
\delta k=\alpha \overline{T w}
$$

where $\alpha$ is proportional to the temperature coefficient, we have

$$
\delta \mathbf{k}=\alpha \mathbf{\alpha} \overline{\mathbf{T}}
$$

This means that using an average temperature and an average reactivity coefficient will underestimate the reactivity effect of temperature feedback unless the factor $\mu$ is taken into account.

The weight factor $\mu$ is the subject of this report.

If one wishes to replace many-group calculation with a few-group calculation, the absorption cross section (say) of one of the few broad groups must be related in some way to the absorption cross sections in the many groups. For example, we might take

$$
\Sigma_{G}^{a}=\sum_{g \in G} \Sigma_{g}^{a} / w_{g \in G}
$$

making the broad group cross section the average of the few groups. Taking the definition to be

$$
\Sigma_{G}^{a}=<\sum_{g \in G} \Sigma_{g}^{a} \phi_{g}>/<\sum_{g \in G} \phi_{g}>
$$


where

$$
r>=\int_{v_{0}} \cdot d v / v_{0}
$$

states that the broad cross section is derived by flux weighting.

And, $\Sigma_{G}^{2}=<\sum_{g \in G} \Sigma_{g}^{a} \phi_{g}^{+} \phi_{g}>/<\sum_{g \in G} \phi_{g}^{+} \phi_{g}>$

states that flux-adjoint weighting is used. If the multigroup case has feedback through some function $f(T)$ with terms of the form

$$
\Sigma_{G}^{a}\left(1+A_{G}^{a} f(T)\right) E_{G}
$$

The relationship between $A_{G}^{a}$ and some weighted sum of $\alpha_{g}^{a}$ will introduce a weight factor. For example, it might be desirable to have

$$
A_{G}^{a}<\Sigma_{G}^{a} \Phi_{G}^{f(T)}>=<\sum_{g \in G} \Sigma_{g}^{a} \alpha_{g}^{a} \phi_{g} f(T)>
$$

in which case, the W.F. might be defined as

$$
\text { M.F. }=\mathbb{N}_{g \in G} \mathrm{~A}_{G}^{a} / \sum_{g \in G} \alpha_{g}^{a}, .
$$


that is, the ratio of the broad group coefficient to the average of the fine groups.

If one wished to reduce the geometric complexity of a problem. (rather than the spectral complexity), we should have some way to maintain the effect of the 3D detail in a (say) 2D mode1. Thus, we could require

$$
\Sigma_{g}^{a}(x, y)=\left\langle\Sigma_{g}^{a}(x, y, z)\right\rangle_{z}
$$

where $<>_{2}$ is an average over $z$ alone, and for the feedback effects we should wish to perhaps balance absorption rates 80 that

$$
\begin{aligned}
& \Sigma_{g}^{a}(x, y) \alpha_{g}^{\alpha}(x, y) \phi_{g}(x, y) f(T(x, y))= \\
& \quad=\left\langle\Sigma_{g}^{a}(x, y, z) \alpha_{g}^{a} \alpha_{g} f(T(x, y, z))\right\rangle_{z}
\end{aligned}
$$

we might then define

$$
\alpha_{g}^{a}(x, y)=\frac{\left\langle\Sigma_{g}^{a}(x, y, z) \alpha_{g}^{a}(x, y, z) \beta_{g}(x, y, z) f(T(x, y, z))>_{z}\right.}{\left.\left.<\Sigma_{g}^{a}(x, y, z) \phi_{g}(x, y, z)\right\rangle_{z} f(<T(x, y, z)\rangle_{z}\right)}
$$

and the W.F. follows as

$$
w F=\alpha_{g}^{a}(x, y) /<\alpha_{g}^{\alpha}(x, y, z)>_{z}
$$


Even if $\alpha_{g}^{a}(x, y, z)$ is space independent, $\alpha_{g}^{a}(x, y)$ will generally be space dependent; this is one of the consequences of trying to maintain the effect of the third dimension in a $2 \mathrm{D}$ representation.

The พ.F. defined by Eq. (0.10) does not use a weighting other than the local flux. There is no reason, however, why an alternate weighting (flux-adjoint) could not be used and indeed there are several reasons for doing so.

In the rormal representation of the multigroup equations, we have both a forward and adjoint formulation. In matrix opej:ator notation we have

$$
\begin{aligned}
& \text { L' }=0 \\
& \mathbf{I}^{+} \cdot \Phi^{+}=0
\end{aligned}
$$

Assume now that the operator I- admits a complete set of eigenvalues, hence

$$
L \cdot \Psi_{n}=\mu_{n} \Psi_{n}
$$

with $\left|\mu_{n}\right|>\left|\mu_{n-1}\right|$ and under reasonable conditions we have, as well

$$
I^{+} \cdot \varphi_{m}^{+}=\mu_{m} \Psi_{m}^{+}
$$

and

$$
\left\langle\Psi_{m}^{+} \Psi_{n}\right\rangle=8_{m n}
$$


Under transient conditions, where (-) satisfies

$$
I \cdot \theta=\frac{\partial \Theta}{\partial t}
$$

we have

$$
\theta(r, t)=\sum_{n=0}^{\infty} a_{n}(t) \Psi_{n}(r)
$$

with $\oplus(x, 0)=\Phi=\Psi_{0}$. Now the determination of the $a_{n}(t)$ is made through use of the adjoint functions

$$
a_{n}(t)=\left\langle\dot{\Psi}_{n}^{+} \otimes(x, t)\right\rangle
$$

It follows then that if we reduce $L(x, y, z)$ to $L(x, y)$, we must eventually wish to preserve the time motion of $a_{0}(t)$ (the dominant time mode related to the dominant time eigenvalue): thus, the reduction must eventually expect that

$$
\begin{array}{r}
\left.<\psi_{n}^{+}(x, y) \oplus(x, y, t)\right\rangle_{x, y}=\left\langle\psi_{u}^{+}(x, y, z) \oplus(x, y, z, t)>_{x, y, z}\right. \\
(0.17)
\end{array}
$$

but this implies that

$$
\begin{aligned}
& \left.\left\langle\psi_{0}^{+}(x, y) \mathcal{L} \cdot \psi_{0}(x, y)\right\rangle_{X, Y}=\Psi_{0}^{+}(x, y, z) L \cdot \Psi_{0}(x, y, z)\right\rangle_{x, y, z} \\
& \text { (0.18) }
\end{aligned}
$$

which defines the operator $\&$ through flux-adjoint weighting. 
For group collapsing identical conclusions can be drawn, except that (for pointwise collapsing) the averaging operator $<$. > is replaced by group averaging.

We shall only deal with spatial W.F.'s.

Thus, we will use flux-adjoint weighting in determining cross sections and feedback coefficients for an "equivalent" ID or 2D system. It is not clear that flux-adjoint weighting guarantees the desired result but it seems probable that no other generally applicable method can guarantee it. We shall use flux-adjoint weighting in determining feedback W.F.'s and shall show that it does preserve many of the multidimensional time phenomena. Applied to the question of xenon feedback stability, these W.F.'s explain the results obtained by Pearce ${ }^{1}$ and Lee. ${ }^{2}$ 
I Definitions and Calculation Methods

It is not difficult to show that in a given state of a reactor (by which we mean given flux, temperature, etc. distributions) the $\Delta k$ content of a feedback mechanism $T(r, z, t)$, which feeds back on the cross section through $a \cdot$ function $f(T(r, z, t))$ and a coeficicient $\alpha(r, z)$ is proportional to the inner product

$$
\left.<\phi^{+}(r, z, t) \alpha(r, z) f(T(r, z, t)) \phi(r, z, t)\right\rangle
$$

In a static one-group model, we have explicitly

$$
\Delta k[T]=\frac{\left\langle\phi_{0}^{2} \alpha f(T)\right\rangle}{\left\langle\Sigma_{a} \phi_{0}^{2}+D\left(\nabla \phi_{0}\right)^{2}\right\rangle}
$$

The definition of a spatial W.F. can be made clear by means of Eq. (1.1). Suppose the original geometry is $(r, z)$ and the final geometry if (r). The inner product in $\mathrm{Bq}$. (1.1) is then over both $r$ and $z$. Initially, let us suppose $h(x, z, t)$ is separable

$$
\phi(r, z, t)=\gamma(x, t) g(z)
$$

then 


$$
\Delta k[T]=\frac{\left\langle\psi^{2} \frac{\left\langle\alpha g^{2} f(T)\right\rangle_{z}}{\left\langle\alpha g^{2}\right\rangle_{z} f\left(\langle T\rangle_{z}\right)} \frac{\left\langle\alpha g^{2}\right\rangle_{z}}{\left\langle g^{2}\right\rangle_{z}} f\left(\langle T\rangle_{z}\right)\right\rangle_{R}}{\left.\psi^{2} \frac{\left\langle\Sigma_{a} g^{2}\right\rangle_{z}}{\left\langle g^{2}\right\rangle_{z}}+(\nabla \Psi)^{2} \frac{\left\langle D g^{2}\right\rangle_{z}}{\left\langle g^{2}\right\rangle_{Z}}+\psi^{2} \frac{\left\langle D(\nabla g)^{2}\right\rangle_{Z}}{\left\langle g^{2}\right\rangle_{Z}}\right\rangle_{R}}
$$

The z-space collapsed values of $\Sigma_{a}, D, \alpha$ and $f$ and $\mathrm{DB}_{2}{ }^{2}$ appear naturally as

$$
\begin{aligned}
& \bar{\Sigma}_{a} \equiv<\Sigma_{a} g^{2}>_{2} /<g^{2}>_{2} \\
& \bar{D} \quad=\left\langle D g^{2}>_{2} /<g^{2}>_{2}\right. \\
& \left.\bar{\alpha} \quad \bar{I}<\alpha g^{2}>_{z} /<g^{1}\right\rangle_{z} \\
& f(\bar{T}) \equiv f(\langle\mathbb{P}) \\
& \left.\mathrm{DB}_{z}^{2}=\left\langle\mathrm{D}(\mathrm{Fg})^{2}\right\rangle_{z} /<\mathrm{g}^{2}\right\rangle_{z}
\end{aligned}
$$

The remaining term is the W.F. associated with $\alpha$ and $f(T)$

$$
\text { W.F. }=\frac{\left\langle\alpha g^{2} f(T)\right\rangle_{2}}{\left\langle\alpha g^{2}\right\rangle_{2} f(\bar{T})}
$$

Eq. (1.4), which may well be time dependent, defining what might be called the instantaneous statistical W.F. (S.W.F.) is what would be determined from perturbation theory; this 
W.F. may be $I$ dependent due to the form of $\alpha(r, z)$ or $f(T(I, z, t))$; it will be time dependent unless $f(T)$ is separable (the separability of $T(r, z, t)$ is not sufficient).

We notice that the S.W.F. $=1$ if $f(T)$ is $z$ independent; that is, a W.F. $\neq 1$ is associated with the fact that the feedback is spatially varying. It is possible then to define an equivalent system where $T$ is space independent and equal to $T_{\text {ave }}$ from the spatially varying case. Here, we have

$$
\Delta k\left(f_{\text {ave }}\right)=\frac{\left\langle\phi_{1}^{2} \alpha\right\rangle f\left(T_{\text {ave }}\right)}{\left\langle\Sigma_{a} \phi_{1}^{2}+D\left(\nabla \phi_{1}\right)^{2}\right\rangle}
$$

and the W.F. associated with $T_{\text {ave }}$ is

W.F. $=\frac{\Delta k(T)}{\Delta k(T a v e)}=\frac{\left\langle\Sigma_{a} \phi_{1}{ }^{2}+D\left(\nabla \phi_{1}\right)^{2}\right\rangle}{\left\langle\Sigma_{a} \phi_{0}{ }^{2}+D\left(\nabla \phi_{0}\right)^{2}\right\rangle} \frac{\left\langle\phi_{0}{ }^{2} \alpha f(T)\right\rangle^{*}}{\left\langle\alpha \phi_{1}{ }^{2}\right\rangle f(T a v e}{ }^{\prime}$

clearly, in a multigroup calculation the far right side of Eq. (1.6) ** becomes quite complicated and means other than spatial integrals become necessary to determine the W.F.

"The functions $\delta_{0}, \delta_{1}$ are solutions of the (in.this case) one-group diffusion equation under the condition $T$ varying or $T$ constant.

For multigroup cases flux-adfoint weighting replaces flux squared weighting. 
Calculational Methods

To determine the W.F. associated with the mechanism $f(T)$, it is necessary to do two calculations at constant power: the type of calculations done determine the type of W.F. found. Although these W.F.'s may be different numerically, if they are used consistently with their definitions, they should predict the same reactor behavior.

\section{A. Perturbation Theory}

A calculation of the S.W.F. is not unreasonable for up to 2 or 3 groups, although some approximations will have to be made in determining the adjoint during transients. In section $v$, we shall consider a two-group example in detail.

\section{B. Equivalent Average Feedback}

This method uses the following procedure. We calculate the eigenvalue for the system at some power level $P_{0}$ with the cross sections altered according to $\alpha(r, z) f(T(r, z))$, and then recalculate the number $\eta$ which corrects the cross sections by $\alpha(r, z) \eta f(T$ ave $)$ and yields the same power level (both systems are just critical at $P_{0}$ ); then,

$$
\eta=\mathbf{W} \cdot \mathbf{F}
$$

In fact, $n$ calculated this way has the same value as that defined by Eq. $(1.6)$. 


\section{Westinghouse Approach}

Westinghouse ${ }^{3}$ makes use of a method similar to the one above. Essentially, they calculate the number $\mu$ which multiplies the average temperature increase above inlet and which yields the same power level; the relationship between the two methods yields.

$$
\eta=E\left(\mu \Delta T_{\text {ave }}+T_{0}\right) / f\left(T_{\text {ave }}\right)
$$

As we shall see later for Doppler feedback, $\eta<\mu$. of course, if $f(T)$ equals $\Delta T$, then $\eta=\mu$.

\section{Semi-Arbitrary Definition}

If, in a point model calculation, the feedback effect of $f\left(T_{\text {ave }}\right)$ is given by an arbitrary relation.

$$
\Delta k_{\text {pt. }}=g\left(T_{\text {ave }}\right)
$$

then the W.F. is still

$$
\text { W.F. }=\Delta k(T) / \Delta k \text { pt. }
$$




\section{E. Method of Eigenvalues}

In a system where many feedback mechanisms are operating and where control rods are moving, the extraction of the separate feedback reactivity effects can be quite complicated. In a heuristic way, we present an approach to determining the amount of $\mathrm{Ak}$ related to some feedback mechanism by a method of eigenvalues. To make the development clear, we use one-group theory.

The partial derivative with respect to a temperature, $T_{i}(r, t)$, (say) of the local feedback is (neglecting second order terms):

$$
\begin{aligned}
\frac{\partial \Delta k_{F B}\left(\phi, T_{i}, \ldots T_{N}\right)}{\partial T_{i}} & =\frac{\alpha \phi^{2} \partial \frac{f_{i}\left(T_{i}\right)}{\partial T_{i}}}{\left\langle\Sigma_{a} \phi^{2}+D(\nabla \phi)^{2}\right\rangle} \\
& \approx \frac{\alpha \phi^{2}\left(f_{i}\left(T_{i}\right)-f_{i}\left(T_{i}-\delta T_{i}\right)\right)}{\left.<\Sigma_{a} \phi^{2}+D(\nabla \phi)^{2}\right\rangle \delta T_{i}}
\end{aligned}
$$

If $\delta T_{i}(r, t)=T_{i}(r, t)-T_{i}(r, t-\Delta t)$, then

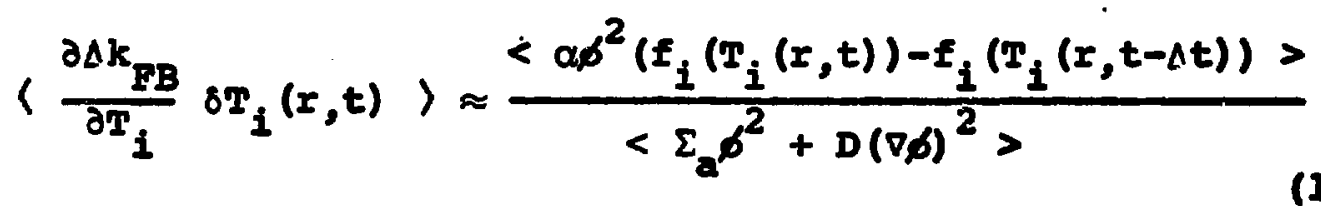

Now, the left side of $\mathrm{Eq}$. (1.11) can be considered to be the amount of $\Delta k$ in the $i^{\text {th }}$ feedback introduced during the time interval $\Delta t$ by the feedback increment $8 T_{i}(r, t)$. 
If we subscript $t_{n}=t, t_{n-1}=t-\Delta t_{n}$, then

$$
\begin{aligned}
\Delta k_{F B_{i}}(t) & \approx \sum_{n}\left\langle\frac{\partial \Delta k_{F B}}{\partial T_{i}} \delta T_{i}(n, n-1)\right\rangle \\
& \approx \sum \frac{\left\langle\alpha \phi^{2}\left(x, t_{n}\right) f_{i}\left(T\left(x, t_{n}\right)\right)\right\rangle-\left\langle\alpha \phi^{2}\left(r, t_{n}\right) f_{i}\left(T\left(x, t_{n-1}\right)\right\rangle\right.}{\left\langle\Sigma_{a} \phi^{2}\left(r, t_{n}\right)+D\left(\nabla \phi\left(x, t_{n}\right)\right)^{2}\right\rangle}
\end{aligned}
$$

where $6 T_{i}(n, n-1)=T_{i}\left(x, t_{n}\right)-T_{i}\left(r, t_{n-1}\right)$

If now $\left\langle 6 T_{i}(n, n-1)\right\rangle$ is small enough and $\lambda\left(t_{j}, T_{i}\left(x, t_{j}\right)\right)=\lambda_{i}(j, j)$ is the system eigenvalue associated with a temperature distribution $T_{i}\left(r, t_{j}\right)$ with all other feedbacks also evaluated at $t_{j}$, then

$\lambda\left(t_{n}, T_{i}\left(x, t_{n}\right)-\lambda\left(t_{n}, T_{i}\left(x, t_{n-1}\right)\right) \approx \frac{<\alpha \phi^{2}\left(x, t_{n}\right) \Delta \varepsilon_{i}{ }_{n}}{\left\langle\sum_{a} \phi^{2}+D(\nabla \phi)^{2}\right\rangle}\right.$

where $\Delta f_{i n}=f_{1}\left(T\left(x, t_{n}\right)\right)-f_{i}\left(T\left(x, t_{n-1}\right)\right)$

and

$$
\Delta k_{E B_{1}}(t)=\sum_{n=1}^{n}\left(\lambda_{i}(n, n)-\lambda_{i}(n, n-1)\right.
$$

This development was suggested by the mothod used by $6.2,4,5$ to calculate Doppler feedback. A.W.F. can be calculated by the mathod of section $D$ : 
II Weight Factors and Xenon Stability

The use of a W.F. in xenon stability analyais is slightIy novel. Its introduction, however, is not only a necessary concomitant to spatial collapsing but explains the paradoxical results obtained by Pearce ${ }^{l}$ and Lee ${ }^{2}$ who neglected to account for the xenon W.F.

Pearce $^{l}$ noticed that the form of the stability criterion for the higher mode instability limit is essentially independent of geometry and thus that the weighted flux level at which higher mode instabilities otart are essentially geometry independent. This result leads to the conclusion that the average flux in $a 3 D$ and id reactor at the instability limit are significantiy different. Iee $^{2}$ observed that when a 3D cubical geometry model was quite unstable the 2D square geometry model was at neutral stability and the ID line model quite stable even though all three modele were at the same power level. These results (of Pearce and Iee) lead to the same paradoxical conclusion that the flux level for the same type of instability (firat axial overtone say) dependa on the degree of detail in the transverse directions. If true, this would imply the imposeibility of spatial separation. A detailed analysis shows that this is not true. 


\section{The 3D yode1}

We take the simplest model to be a one-group model in a bare homogeneous core:

$$
\begin{aligned}
\frac{1}{v} \frac{\partial \phi}{\partial t} & =D \nabla^{2} \phi+\left(v \Sigma_{f}-\Sigma \Sigma_{a}-\gamma \phi-\sigma_{x} X\right) \phi \\
\frac{\partial I}{\partial t} & =y_{I} \Sigma_{E} \phi-\lambda_{I} I \\
\frac{\partial X}{\partial t} & =-\lambda_{X} X+y_{x} \Sigma_{I} \phi+\lambda_{I} I-\sigma_{x} X \phi
\end{aligned}
$$

with equilibrium $\beta_{0}, I_{0}, x_{0}$, and $\gamma$ is the $f l u x$ coefficient of reactivity.

Iinearizing these equations and taking a Laplace transform, we find:

$$
\begin{aligned}
& D \nabla^{2} \tau+\left(\nu \Sigma_{f}-\Sigma_{a}-\gamma \delta_{0}-\sigma_{x} x_{0}\right) \Psi+\left(\frac{1}{v} s+\gamma \phi_{0}\right) \tilde{y}-\sigma_{x} \delta_{0} \tilde{X}=0 \quad \text { (2.2a) }
\end{aligned}
$$

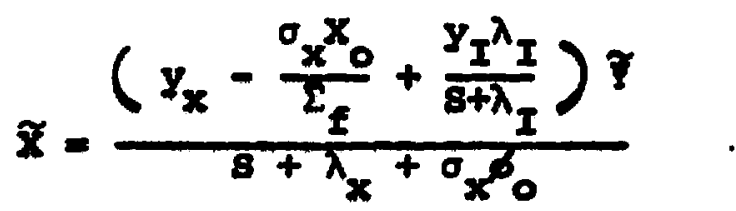

The operator

$$
\text { L. }=D \nabla^{2}+\left(v \Sigma_{f}-\Sigma_{a}-\gamma \delta_{0}-\sigma_{x} x_{0}\right)
$$

i. a sturm-Iiouville operator admitting a complete set of 
orthogonal modes $g_{n}(x, y, z)$ with eigenvalues $D_{\mu_{n}}{ }^{*}$; expanding . $\Psi$ in these modes as

$$
\tilde{y}=\Sigma a_{n}(s) g_{n}(x, y, z)
$$

taking inner products with weight $g_{n}$ and neglecting modal interaction we find the characteristic equation to be:

$$
\begin{aligned}
0= & \left.<g_{n}^{2}>\left(\frac{1}{v} s+\mu_{n}^{2} D\right)+\gamma<\phi_{0} g_{n}^{2}\right\rangle+ \\
& \left.+<\sigma_{x} \Sigma_{f} \delta_{0} g_{n}^{2} \frac{\left(y_{x}-\frac{\sigma_{x} X_{0}}{\Sigma_{f}}+\frac{y_{I} \lambda_{I}}{s+\lambda_{I}}\right)}{s+\lambda_{x}+\sigma_{x} \sigma_{0}}\right\rangle
\end{aligned}
$$

It is not practical to analyze Eq. (2.3) in general, but in the low and high flux limits we find the following stability criteria.

Low Flux Iimit:

If $\sigma_{x} \delta_{0}<\lambda_{x}$, then the system is marginally stable whon

$$
\left.t_{n}=\frac{\left\langle\phi_{o} g_{n}^{2}\right\rangle}{\left\langle g_{n}^{2}\right\rangle}=\frac{\lambda_{x}\left(\lambda_{x}+\lambda_{x}+\mu_{n}{ }^{2} D v\right)}{v\left(y \sigma_{x}-\gamma\right)}\right\rangle 0
$$

"In 3D geometry we really should have subscripted $g$ and $\mu^{2}$ with 3 ubscripts, these are suppressed for convenience. 
19

High Flux Limit:

If $\sigma_{x} \phi_{0}>\lambda_{x}$, then the system is marginally stable when

$$
\gamma_{n}^{\Phi}=\Sigma_{f} Y_{I}-\mu_{n}^{2} D>0
$$

2. The 1D Model

By analogy to the 3D model, we can write down the ID model at once (let $\left.\phi^{1 D} \equiv \phi_{1}, \phi^{3 D}=\beta_{3}\right)$

$\frac{\overline{1}}{v} \frac{\partial \phi_{1}}{\partial t}=\frac{\partial}{\partial x}\left(\bar{D} \frac{\partial \phi_{1}}{\partial x}\right)-\overline{D B_{1}{ }^{2} \phi_{1}}+\left(\overline{\nu \Sigma_{f}}-\overline{\Sigma_{a}}-\bar{\gamma} \phi_{1}-\bar{\sigma}_{x} X_{1}\right) \phi_{1} \quad$ (2.5)

The general method of spatial collapsing used here' is fluxadjoint (in one-group, flux squared) weighting; hence, (in this simple case).

$\frac{\bar{I}}{v}=\left\langle\phi_{3}^{2} \frac{1}{v}\right\rangle_{y, z} /\left\langle\phi_{3}^{2}\right\rangle_{y, z}$

(2.6)

$\bar{D}=\left\langle b_{3}{ }^{2} D\right\rangle_{y, z} /\left\langle b_{3}{ }^{2}\right\rangle_{y, z}$

(2.7)

$\left.\bar{\nu}_{f}\left(\Sigma_{a}\right)=\left\langle\phi_{3}^{2} v \Sigma_{f}\left(\Sigma_{a}\right)\right\rangle_{y, z} /<\phi_{3}^{2}\right\rangle_{y, z}$

(2.8)

$\left.\left.\bar{\gamma}=\left\langle\gamma \beta_{3}{ }^{3}\right\rangle_{y, z} /<\phi_{3}{ }^{2}\right\rangle_{y, z}<\phi_{3}\right\rangle_{y, z}$

(2.9) 


$$
\begin{aligned}
& \left.\left.\bar{\sigma}_{x}=\left\langle\sigma_{x} \phi_{3}{ }^{2} x_{3}\right\rangle_{y, z} /<\phi_{3}{ }^{2}\right\rangle_{y, z}<x_{3}\right\rangle_{y, z} \\
& \left.\overline{D B_{1}{ }^{2}}=-\left\langle\phi_{3} D\left(\frac{\partial^{2} \phi_{3}}{\partial y^{2}}+\frac{\partial^{2} \phi_{3}}{\partial z^{2}}\right)\right\rangle_{y, z} /<\phi_{3}{ }^{2}\right\rangle_{y, z}
\end{aligned}
$$

It is in the definitions for $\bar{\gamma}$ and $\bar{\sigma}_{\mathbf{x}}$ that the weight factors enter, since we may say

$$
\begin{aligned}
& \text { Xenon W.F. }=\text { X.W.F. } 1=\frac{\left\langle\phi_{3}^{2} x_{3}\right\rangle_{y, z}}{\left\langle\phi_{3}^{2}\right\rangle_{y, z}\left\langle x_{3}\right\rangle_{y, z}}(2.12 a) \\
& \text { Temperature W.F. } \quad=\frac{\left\langle\phi_{3}^{3}\right\rangle y_{2}}{\left\langle\phi_{3}^{2}\right\rangle_{y, z}\left\langle\phi_{3}\right\rangle_{y, z}}(2.12 b)
\end{aligned}
$$

In the xenon equation we have on straight averaging*

$$
\dot{x}_{1}=y_{x} \bar{\Sigma}_{f} \phi_{1}+\lambda_{I} I_{1}-\overline{\bar{\sigma}}_{x} x_{1} \phi_{1}-\lambda_{I} I
$$

where

*straight averaging is convenient; if some other averaging
were used, Eq. (2.10) and Eq. (2.13c) would be changed to
reflect such differences. Physically, however, it seems
"right" to straight average xenon concentrations, tempera-
tures, etc., since then the calculated power distribution
should, reflecting the average feedback, be the average power. 


$$
\begin{gathered}
\bar{\Sigma}_{f}=\left\langle\Sigma_{f} \delta_{3}\right\rangle_{y, z}\left\langle<\phi_{3}\right\rangle_{y, z} \\
\bar{\sigma}_{x}=\frac{\left\langle\sigma_{x} x_{3} \delta_{3}\right\rangle_{y, z}}{\left\langle\sigma_{3}\right\rangle_{y, z}\left\langle x_{3}\right\rangle_{y, z}} \equiv \sigma_{x} X \cdot W \cdot F_{\cdot 2}
\end{gathered}
$$

Since the iodine equation is linear, it presents no problem independently of the type of averaging used.

\section{Interpretation}

a) $\phi_{1}$ would normally be assumed to be the average over $y, z$ of $\alpha_{3}$, hence if

$$
\begin{aligned}
& \phi_{1}=\phi_{1 p} g_{1}(x) \\
& \phi_{3}=\phi_{3 p} g_{3}(x, y, z)
\end{aligned}
$$

we have

$$
\phi_{1 p}=\phi_{3 p}<g_{3}>_{x, y, z^{\prime}}<g_{1}>_{x}
$$

if $g_{3}=\sin \frac{\pi x}{\bar{B}_{1}} \sin \frac{\pi y}{\bar{B}_{2}} \sin \frac{\pi z}{\bar{H}_{3}}$ and $g_{1}=\sin \frac{\pi x}{\bar{B}_{1}}$, as Pearce 1 assumed, then

$$
\delta_{1 p}=\left(\frac{2}{\pi}\right)^{2} \delta_{3 p}
$$


This result says that the peak 1D flux is only about $40 \%$ of the $3 D$ value.

b) The temperature and xenon W.F.'s appear in a natural way due to the flux-adjoint weighting, and increase the mean values of $\gamma$ over that used in the 3D case; the temperature W.F. for example, has the (bare core) value:

$$
\text { T.W.F. }=(4 / 3)^{2}
$$

while in the low flux limit the xenon W.F. has the same value.

c) In the bare core, the ratio of the critical 3D and ID weighted fluxes (c.f. Eq. (2.4a) alone yield

$$
\frac{\Phi_{n}^{3 D}}{{ }_{n}^{1 D}}=\left(\frac{8}{3 \pi}\right)^{2} \frac{\delta_{3 p}}{\delta_{1 p}}
$$

Pearce and Lee point out (in effect) that if both geornetries operate at the same power level, then Eq. (2.15) is valid and

$$
\frac{n^{3 D}}{n^{1 D}}-\left(\frac{4}{3}\right)^{2}
$$

This would indicate that if the 3D system was barely stable the ID would be quite stable. The correct spatial collapsing 
method shows, however, that what must be considered is (at low flux)

$$
\frac{\left(y \sigma_{x}-\gamma\right) \Phi_{n}^{3 D}}{\left(y \bar{\sigma}_{x}-\bar{\gamma}\right) \Phi_{n}^{1 D}}
$$

and that this ratio is indeed unity. The same result is seen at the high flux limit where $\gamma_{n}^{\Phi_{n}}{ }^{3 D} / \bar{\gamma}_{n}{ }^{1 D}=1$. Thus, the paradoxical results of Pearce and Lee disappear if. a consistent spatial collapsing scheme is used. This result is in itself somewhat paradoxical, since a flux-adjoint weighting should preserve the fundamental mode time eigenvalue but not the higher modes. This is resolved by noting that the higher mode eigenvalues are in all cases associated only with the " $\mathrm{X}$ " direction, and the collapsing is in the $y, z$ directions. It seems unlikely that these W.F.'s will preserve the exactly correct behavior at all power levels but it seems clear that far better agreement is obtained by including the W.F.'s than by excluding them.

d) The development shows that the xenon cross section must be weighted differently in the neutronics equation and the xenon equation itself. This result cannot be avoided. No code (as far as we know) allows for the introduction of xenon W.F.'B.

e) The fission cross section $\Sigma_{f}$ is weighted differently in the xenon equation than in the neutronics. If 
fission cross section feedback exists, this implies that different feedback W.F.'s are needed in determining the power (which enters the xenon and temperature equations) than are used in determining the flux (in the neutronic equations). No code (as far as we know) allows for this effect which can be considerable in systems with high peak/average power values. 
III Weight Factors and Inlet Coolant Temperature Changes A general form for $\Sigma_{a}$ (say) which accounts for feedback effects can be written as:

$$
\Sigma_{a}=\Sigma_{a}^{0}\left[1+\sum_{i} \alpha_{i}\left(T_{i}(x, y, z, t)-T_{I N}\right)+\ldots\right]^{*}
$$

When a reactor is at zero power, all components have the same temperature equal to the inlet coolant temperature (TIf). If this inlet temperature changes, the core components undergo a temperature transient which may be calculated by the usual temperature equations but with zero source. For a step change in inlet temperature, one sees a wave of moderator temperature move down the channel and a gradual relaxing of the clad and fuel temperature to the new temperature.

As before, no problems arise in a 3D calculation but spatial collapsing introduces a completely new set of weight factors which pertain only to changes in inlet coolant temperature. Suppose the new base temperatures are labelled $T_{j}^{0}(x, y, z, t)$ (for constant inlet temperature all $\left.T_{j}^{0}=T_{I N}\right)$, then we may rewrite Eq. (3.1) as

*Fuel temperature effects appear through $\left(\sqrt{T_{f}}-\sqrt{T_{I r}}\right)$, but this does not affect the analysis; the dots atand, in general, for all other forms of feedback. 


$$
\begin{aligned}
\Sigma_{a}= & \Sigma_{a}^{0}\left[I+\sum_{i} \alpha_{i}\left(T_{i}(x, y, z, t)-T_{i}^{0}(x, y, z, t)\right)\right. \\
& \left.+\sum_{i} \alpha_{i}\left(T_{i}^{0}(x, y, z, t)-T_{I N}\right)+\ldots\right]
\end{aligned}
$$

While $T_{i}(x, y, z, t)-T_{i}{ }^{0}(x, y, z, t)$ is proportional to the power, $T_{i}{ }^{0}(x, y, z, t)-T_{I N}$ is not; hence, in spatial collapsing, a different weight factor appears associated with inlet temperature changes. This factor is nearly unity, especially if no radial orificing exists; but independently of orificing, the axial W.F. associated with $T_{i}{ }^{0}$ has the form

$$
A x \text {.W.F. } \approx 1-\frac{\Delta T_{I I} \sin (2 \pi v t / H)}{T_{I I}^{\circ} 2 \pi\left(1+\frac{\Delta T_{I I}}{T_{I I}^{0}} \frac{v t}{H}\right)}, v t<B
$$

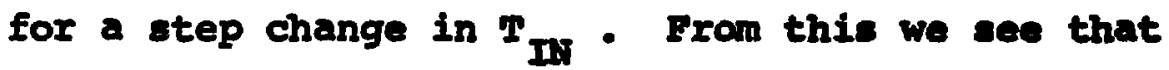

$$
1-\frac{\Delta T_{m}}{2 \pi T_{m}^{0}}<A_{x} W<1+\frac{\Delta T m_{H}}{2 \pi T_{m}^{0}}
$$

\footnotetext{
*veglecting slower effects $\mathrm{T}_{i}^{0} \approx \mathrm{T}_{\mathrm{IX}}+\Delta \mathrm{T}_{\mathrm{IN}} \mathrm{s}\left(\frac{v t}{\mathrm{H}}-x\right)$ and $\left\langle\phi^{2} \mathrm{~T}_{i}^{0}\right\rangle /<\mathrm{T}_{i}^{0}>$ yielda Eq. (3.3).
} 
Even for $20 \%$ changes in $T_{\mathrm{Is}}^{\circ}$, we see from Eq. (3.4) that only 3X changes in Ax.W.F. occur; because of this we expect that we may neglect the W.F. for inlet changes and call it unity. In radially collapsed form, Bq. (3.2) becomes

$$
\begin{aligned}
\Sigma_{a}(x, t)=\Sigma_{a}^{0}(x)[1 & +\sum_{i} \alpha_{i} W_{\cdot} F_{\cdot_{i}}\left(T_{i}(x, t)-T_{i}^{0}(x, t)\right) \\
& \left.+\sum_{i} \alpha_{i}\left(T_{i}^{0}(x, t)-T_{I D}\right)+\ldots\right]
\end{aligned}
$$

In this approximation 1 W.F. appears associated with the increase in temperature above the local base temperature, but no K.F. appeare associated with changes in the base temperatures due to changes in the inlet coolant tomperature.

These results have some significance with relation to pipeline breaks. A break in the core coolant line on the exit eide produces an increase in coolant velocity which affects the temperature increase above inlet; hence, in collapsed geometry a fallure to account for the radial moderator V.F. can mean a $50 \%$ decrease in the amount of reactivity inserted into the syetem (if the moderator coefficient is nogative). Alternatively, if the break occure in the secondary coolant pipelines, then the core inlet temperature will be affected and the M.I.' 's do not onter into dotermining the reactivity input.

To our knowledge, no codes allow for these effects. 
IV Partial Weight Factors

Although implicit in the analysis already carried out, we wish to make clear that the W.F.' 's associated with feedback will generally be different for the $\Sigma_{a}, \Sigma_{E}$ and $D$ components of that feedback. The 3D apatial distribution of $\frac{1}{\Sigma_{a}} \frac{\partial \Sigma_{a}}{\partial r_{1}}=a_{a, 1}$ will not be apatially uniform (except in a multigroup model) due to apatial spectral variation. Thus, $w_{a, 1}, W_{E, 1}$, and $w_{f_{, i}}$ can be considerably different. This can be seen in rables 1 and 2 . 


\section{Table 1}

Partial Radial Doppler Weight Factors for a BKR

Core statue

$2.5 \%$ Rod Drop

Configuration

$3.8 \%$ Rod Drop

Configuration

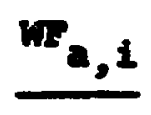

2.509

2.831

2.193

2.586

4.91
$W_{E, 1}$

${ }^{N, 1}$

Doppler

nT.

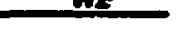


Table 2

Partial Radial Doppler and Noderator Weight Factore

for a Typical $B$ and $W$ PWR core

\begin{tabular}{|c|c|c|c|c|c|c|c|c|}
\hline \multirow[t]{2}{*}{$\begin{array}{c}\text { Core } \\
\text { Conficuration }\end{array}$} & \multicolumn{4}{|c|}{ Doppler W. Fis } & \multicolumn{4}{|c|}{ voderator uerie } \\
\hline & $a_{a, 1}$ & $\alpha_{E}$ & a. & rotal & $a_{a, j}$ & $\alpha_{E, j}$ & $a_{D, j}$ & Total \\
\hline 1000 swt & 1.247 & 1.424 & .7783 & 1.294 & 1.327 & 1.321 & .8105 & .7414 \\
\hline
\end{tabular}

The moderator W.F.'" lese than unlty have to do with locally positive and negative modarator reactivity coefficiente. 
v Doppler Weight Factors

The balance of this report will concern the calculation of static and dynamic Doppler Weight Factors (D.W.F.), theix relationship to other reactor parameters, the degree of $(x, z)$ separability and the usefulness of such D.W.F.'s in less than 3D transient analysis. Along the way, we shall have reason to introduce a series of special W.F.'s which associated with the feedback mechanisms allow a direct $(r, t)$ calculation at the $(x, z, t)$ fuel hot spot. Comparison between $(r, t),(z, t)$ and $(r, z, t)$ calculations show excellent agreement between peak fuel enthalpy (core hot spot), even though the normal $(x, z, t)$ D.W.F. is not separable.

The Instantaneous Doppler Weight Factor (D.W.F.) is defined by an equation such as Eq. (1.4), i.e.

Radial D.W.F. $=\frac{\left\langle\alpha(x) \phi^{2}(x, t)\left(\sqrt{T_{E}(x, t)}-\sqrt{T_{I}}\right)\right\rangle}{\left.\left\langle\alpha(r) \phi^{2}(r, t)\right\rangle\left(\sqrt{\left\langle T_{E}(r, t)\right.}\right\rangle-\sqrt{T_{I}}\right)}$

Axial D.W.F. $=\frac{\left\langle\alpha(z) \phi^{2}(z, t)\left(\sqrt{T_{f}(z, t)}-\sqrt{T_{I N}}\right)\right\rangle}{\left\langle\alpha(z) b^{2}(z, t)>\left(\sqrt{\left\langle T_{f}(z, t)\right.}\right\rangle-\sqrt{T_{I N}}\right)}$ 


\section{A. Static Doppler Weight Factors}

In this section we calculate and compare the W.F. defined by Eq. (5.0a) and the equivalent radial W.F. defined by Eqs. (1.7) and (1.8); we shall see that the perturbation theory result (Eq. (1.4)) and the method of equivalent average feedback (Eq. (1.7)) are always very close to each other, while the Westinghouse definition (Eq. (1.8)) deviates markedIy for high (relatively speaking) fuel temperatures. * These calculations are done for a series of PWR and BWR cores at low, medium, and high power under various conditions but generally when the core is in a configuration from which a central rod of various worths has been removed. The results are shown in Tables 3 and 4.

*This does not indicate error but reflects the difference in definition. 
Table 3

A Comparison of Variously Calculated Radial DHF's

Reactor Power Nut

\begin{tabular}{|c|c|c|c|}
\hline Rod Worth & S.W.E. & $n$ & $\underline{\mu}$ \\
\hline .03666 & 8.472 & 8.407 & 8.549 \\
\hline .02461 & 5.839 & 5.805 & 5.868 \\
\hline .00961 & 2.300 & 2.297 & 2.303 \\
\hline .00377 & 1.356 & 1.356 & 1.357 \\
\hline .00130 & 1.064 & 1.065 & 1.070 \\
\hline .00200 & 1.084 & 1.087 & 1.094 \\
\hline $.00444^{\circ}$ & 1.323 & 1.360 & 1.3995 \\
\hline .00663 & 1.767 & 1.859 & 1.975 \\
\hline .00998 & 2.640 & 2.731 & 3.082 \\
\hline .01519 & 3.849 & 3.864 & 4.702 \\
\hline .000994 & 4.354 & 4.369 & 5.590 \\
\hline .00182 & 4.391 & 4.387 & 5.622 \\
\hline .00127 & 5.197 & 5.160 & 6.556 \\
\hline .00122 & 1.054 & 1.055 & 1.066 \\
\hline .00182 & 1.063 & 1.067 & 1.081 \\
\hline .00260 & 1.087 & 1.098 & 1.118 \\
\hline .00369 & 1.144 & 1.166 & 1.207 \\
\hline
\end{tabular}


Table 4

A Comparison of Various Radial D.W.F.'s in situations reading to rarge values

Power, lewt

27.5

500
$\underline{S_{2} H_{2}}$

10.43

11.60

15.34

18.07

10.55 $n$

10.36

12.59

25.33

28.24

10.50
11.

10.63

11.86

15.79

18.69

14.70 
It is clear that the statistical W.F. and the Equivalent Eeedback values always agree quite closely; in no case of the few hundreds of cases considered did they differ by more than 8\%. The $\mu$ W.F., however, is clearly different; at low powers (even with relatively large peak/averages) $\eta \approx$ S.W.F. $\approx \mu$, but at higher powers as the peak/average fuel temperature increase above inlet becomes large, the value of $\mu$ becomes considerably larger than $\eta$ or S.W.F. This is not to say that $\mu$ is incorrect; indeed, if it is used in a calculation consistently with its definition, it should yield correct answers. In any event, since we choose to accept the definition of a W.F. as a number (or function) which multiplies the feedback coefficient rather than something which multiplies a temperature rise, we shall not consider the $\mu$ weight factor for the rest of this section.

Because of the closeness of $n$ and S.W.F. and the (relative) simplicity of calculating the s.W.F. over that of calculating $\eta$ (one eigenvalue calculation versus two), we shall assume that the S.W.F. can be used to accurately relate a ID model to a $2 D$ or $3 D$ model. 


\section{B. Predicting the Transient Doppler Weight Factor}

In a one-neutron energy group calculation it is possible to evaluate the integrals in Eq. (5.0) without too much difficulty. For a multi-energy group model, however, the adjoint function, in a case with feedback, becomes much more difficult to calculate and recourse to other means of predicting the D.W.F. are desirable."

1.) One of the first attempts was suggested by G.E. ${ }^{5,6}$; they proposed that the following relation is a valid one:

$$
\frac{P / A-1}{D \cdot W \cdot F \cdot-1}=C
$$

where $: / A$ is the peak/average power, D.W.F. the Doppler W.F. and $c$ is a constant. In Ref. $6, \mathrm{pp} .6,7$, the value of $c$ was taken as 3 . In Ref. 5 (two years later), it is taken as 2.36 (more optimistic). Since the original data (as presented in Ref. 6) fitted $c \approx 3$ quite well, it is not clear why the number was reduced. This minor point is not of great importance since it is clear that $C$ is not constant.

In the simplest possible model of a core of unit length with unit flux, except for a region of length $\varepsilon$ where the

* In a subsequent portion of this report, we shall make use of an approximate two-group adjoint to calculate $(r, z)$ D.W.F.'s during a transient. 
flux is $1+A$, we find that Eq. (5.1) yields

$$
C=\frac{1-\epsilon+\varepsilon(1+A)^{2}}{\epsilon\left[(1+A)^{2}-1\right]}
$$

but for $E \rightarrow O$ at constant $A, C \rightarrow \infty$; while for $A \rightarrow \infty$ at constant $\varepsilon, C \rightarrow 1$; and for $\epsilon A$ constant with $A \rightarrow \infty, C \rightarrow 1$ also. Only if $E A$ is constant for $A$ small and $E A^{2}$ constant for A large will $c$ be constant; clearly, $C$ is not likely to be constant.

In Fig. 1 we plot some of the 27.5 Mwt results for PWR/BWR cores. The lines simply connect the points derived from a given core radial model with variable central rod worths. In Fig. 2, which is more closely related to BWR rod drop analysis, we see that having an unrodded annulus surrounding the central rod leads to the interesting siturtion that for large annuli the value for $c$ becomes slowly varying. The reason for this is that in the case of a large unrodded annulus, the central rodded region does not play a large part in determining the peak/average or the W.F. The multi-valued nature of the curve in Fig. 2 is, in 1tself, interesting, since it states that the $1 \%$ rod dropped with a $13 \mathrm{~cm}$ annulus is more dangerous than the same worth rod dropped with a $48 \mathrm{~cm}$ annulus (assuming both such systems were equally critical, before the rod dropped, which is not likely). 
While we can reasonably state that exceft for special situations the value of $C$ is not constant from one static calculation to another, it is possible that $c(t)$ for a given reactor could have limited variation during a traneient.

The exact method of establishing the model of, and analyzing the rod drop accidents in, a BWR is discusead in detail in Rod Drop and SCRAM in Boiling water Reactors? Fig. 3 shows the variation of $\mathrm{C}$ for a radial mock-up of the 2.5\% BWR rod drop accident. While the response (in this case) is bounded by $c \approx 3$ the variation of $c$ is too large to accept a relation such as Bq. (5.1). Fig. 3 show that $C$ and the D.W.F. undergo a somewhat similar transients Fig. 4, which describes a small worth rod ejection in a typical $B$ and $W$ PWR core, shows that while $C$ undergons a large transient, the D.W.F. remains nearly constant. These results demonstrate that $c$ is a poor correlate for the D.W.F.

2.) Another suggestion by 6.E. was that the Axial D.W.E. (which is needed when doing an $(x, y, t)$ or $(x, t)$ calculation) could be related to a quadratic in the average fuel temperature rise. As we shall show, this suggestion has merit, particularly wo in that it led to the discovery of the "special" axial D.W.F. Which permits the $(x, t)$ calculation to be done at the axial hot spot.

The cross sections and temperature coefficients that enter the $r, z$ calculation were collapeed to yield axial input data. The averagn radial D.W.F. (varying for the various coefficients) used was 3.5 which, as can be seen 
from Fig. 3, is reasonably valid between 0.9 sec and 2.0 sec into the trancient.

The sane $2.5 \%$ rod drop aceidant was calculated fall of these trancients are done with uph unlese otherwise indicated) in axial geometry and an edit of the axial D.W.F. with time is shown in Fis. 5. Figure 6 bhows the cans calculations replotted againot the axial average fuel temparature Incrosse above inlet. These resulte show a downward dished guadratic (on sverage) with a ohaxp upward high ordar toxm (4th or 5th) eftect as the scuny takes effect. nonotheless, if it is acceptoble to dieregard the eariy rise (which occurs with almost no temperature effects) frem 1.25 to 2.85, the reat of the Ax.D.W.F. can be correlated with a relatively simple, although not a purely quadratic, polynemial in average fuel temperature rise. since the average axial and radial fuel temperature increases should be the san for noarly so) if the epatial collapeing is succeseful the ame function my be introduced in the $(x, t)$ calculation in terme of the radial average fuel temperature rise. 


\section{c. Radial calculations at the axtal peak sunl reapere- tyros}

In the normal reduction of an $(x, z, t)$ goometzy to $(x, t)$, - straight average over 2 is usulliy porformad in the feedback (thermal-hydraulic) equations. Thus, the fuel temperature we calculate is - presumably - the axtal average at $(x, t)$. The definition of D.H.F. by means of equations such as Eq. (5.0) is consistent with this, since the avarage temparature appeare in the denominator. Figure 5 shows that the axial D.M.F. is far from constant during a rod drop accidant. This undesirable result makes it neceseary to tind and insert a fitted function tor axial D.M.F. In terms of some variable casily calculable duxing an $(x, t)$ calculation. Even though such - function is deducible, the resulting $(x, t)$ calculation yields (even if fully aceurate) only the axial average values of the fuel tengerature. The subsequent determination of the peak temperatures requires an auxiliery series of sssumptions and calculotions.

We now propose a method of making a dixect caleulation at the axial peak value of the turl temperature. That lo, the peak temperature in the $(x, t)$ calculation will be automatically the hot spot of the core.

Consider a sirple fuel temperature equation such as is used in wwichs

$$
\frac{\partial a_{E}(x, z, t)}{\partial t}=\operatorname{cP}(x, z, t)-\lambda\left(x_{g}(x, z, t)-x_{c}(x, z, t)\right)
$$


where $\mathrm{B}_{\mathrm{f}}$ is the fuel enthalpy, $\mathrm{T}_{\mathrm{f}}$ the fuel temperature, and Te the clad temperature. During a transient the pak fuel enthalpy in the core will oceur at axial location $z_{p}(t)$. The power at this location can be written as

$P(x, t)=P\left(x, z_{p}(t), t\right)=\Sigma_{f}\left(x, z_{p}(t), t\right) \delta\left(z_{p}(t)\right) \&(x, t)$

sines $f\left(z_{p}(t)\right)$ is a simple, time-depandent coefficient, w absorb it into $S(x, t)$ to yields

$$
r(x, t)=f\left(x_{p}(t)\right) S(x, t)
$$

similarly, the socond order affects of fendback on $\varepsilon_{f}$ can be abeorbed

$\varepsilon_{E}^{0}\left(1+a I_{E}\left(x, z_{p}(t), t\right)+\ldots\right)=\varepsilon_{\varepsilon}^{0}\left(1+a g_{E}^{P}(x, t)+\ldots\right)$

$$
\text { - }
$$

where $g_{f} P(x, t)$ is the radial distribution of the susl tmporature at the pakk axial pooktion at "t". The other terme take on whatever values thoy chould hove at the peak suel enthalpy postion. Inxee difficulties present thenealves firatiy. the coolant temperature has the structure

$$
\frac{\partial z_{c}}{\partial t}+v \frac{\partial s_{c}}{\partial s}=c_{1} P+\lambda_{2}\left(x_{2}-s_{c}\right)
$$


Wherever the fuel peaks we replace Eg. (5.6) by

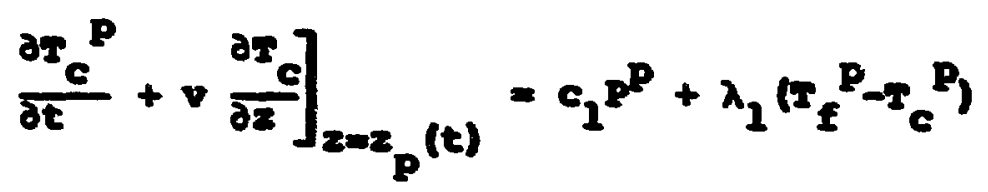

where the super " $p$ " indicates that the variable is evaluated at the axial location of peak fuel enthalpy. we replace the spatial derivative by

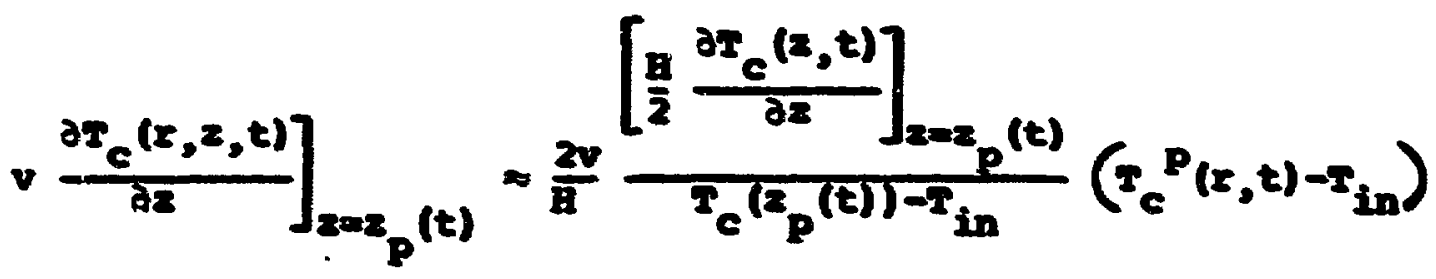

The velocity confficient:

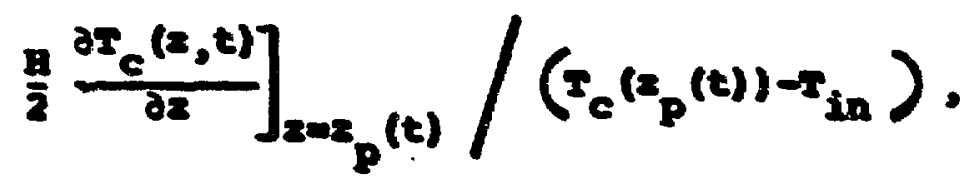

way be calculated in a $(z, t)$ calculation.

Although the uaval afproach is to neglect entirely the heat transier to clad or coolant in bin rod drop calculation (thus, the value of this "velocity factor" is not important from this viewpoint), nonethelese, we shall see from actual calculations that it has only a wak effect 
on the peak fuel enthalpy during $3.8 \%$ rod drop as long as moderator feedback is neglected. The detailed shape of this factor is not simple but it can be chosen so as to yield upper or lower bounds on the moderator temperature increase.

The second difficulty lies in the interpretation of the total reactor power in a calculation of this sort. Iypically, we have:

$$
P_{Y}(t)=2 \pi x \int_{0}^{R} d z \int_{0}^{R} r \Sigma f(x, z, t) d r
$$

assuning

$$
\phi(x, z, t)=\delta(z, t) \phi(x, t)=\phi_{z} \phi_{R}
$$

We have, neglecting 2 variations of $\Sigma_{f}=$

$$
\begin{aligned}
& P_{T}(t)=2 \pi x \int_{0}^{A} \delta(z, t) d z \int_{0}^{R} \Sigma_{f} \delta(x, t) x d x
\end{aligned}
$$

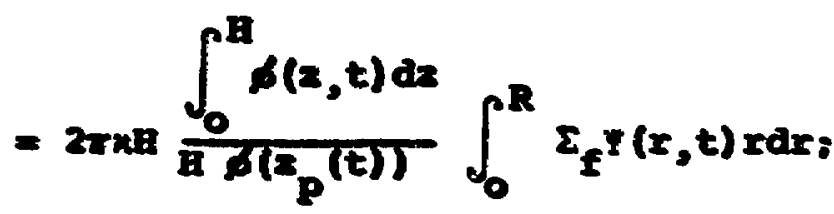

in the calculational process we hove from a $(x, t)$ calculation the "value to average" factors 


$$
p_{E}(t)=H \delta\left(z_{P}(t)\right) / \int_{0}^{H} \phi(z, t) d z
$$

while from the $(r, t)$ calculation we find the power value

$$
P=2 \pi x H \int_{0}^{R} \Sigma_{f} Y(r, t) r d r,
$$

the total power is then

$$
\mathbf{P}_{\mathbf{T}}=\mathbf{P} / \mathbf{P}_{\mathbf{E}}(t)
$$

The total power is used in the $(r, t)$ calculation just twice, once in determining the equilibrium and when determining when the high power trip occurs. Thus, it is necessary to do the $(z, t)$ rod drop and edit $p_{f}(t)$ for use in the $(r, t)$ calculation.

The third difficulty lies in the flux equation itself. In a simplified way we sketch the development of the calculation of $y(x, t)$. In one group, we assume Eq. (5.9) is valid: hence, we have

$$
\frac{\partial \phi_{z} \phi_{R}}{\partial t}=\phi_{R} D \nabla^{2} \phi_{z}+\phi_{z} \nabla \cdot D \nabla \phi_{R}+\left(\nu \Sigma_{f}-\Sigma_{a}\right) \phi_{z} \phi_{R}
$$

* In order to secure accurate results, several iterations may be necessary to secure converged (usually $2(z, t)$ and $2(x, t)$ ) calculations. 
The spatial collapsing scheme uses flux square weighting: hence, eliminating the $z$ coordinate we have:

$$
\left.\frac{\left.\partial<\phi_{z}^{2}\right\rangle \phi_{R}}{\partial t}=\left\langle\phi_{z}^{2}\right\rangle \nabla \cdot D \nabla \phi_{R}+\left[\left\langle\left(\nu \Sigma_{f}-\Sigma_{a}\right) \phi_{z}^{2}\right\rangle+D<\phi_{z} \nabla^{2} \phi_{z}\right\rangle\right] \phi_{R}
$$

if we let $Q$ stand for any $\Sigma$ then we choose

$$
Q=0^{0}\left[1+a_{1} T+a_{2}\left(\sqrt{T_{E}+T_{0}}-\sqrt{T_{0}}\right) \text { etc. }\right]
$$

$\left\langle\alpha \phi_{z}^{2}\right\rangle=Q^{\circ}\left[\left\langle\phi_{z}^{2}\right\rangle+\alpha_{1}\left\langle\phi_{z}^{2} P+\alpha_{2}<\phi_{z}{ }^{2}\left(\sqrt{T_{f}+T_{0}}-\sqrt{T_{0}}\right)\right\rangle+\ldots\right]$

Now we make the same sort of approximation as in Eq. (5.7).

$$
\frac{\left\langle b_{z}^{2} T\right\rangle}{\left\langle b_{z}^{2}\right\rangle} \approx \frac{\left\langle b_{z}^{2} T(z, t)\right\rangle}{\left.\left\langle b_{z}^{2}\right\rangle T(z, t)\right)} T_{p}^{P}(r, t)
$$

Dividing both sides of Eq. (5.12) by $\left\langle\alpha_{2}^{2}\right\rangle$

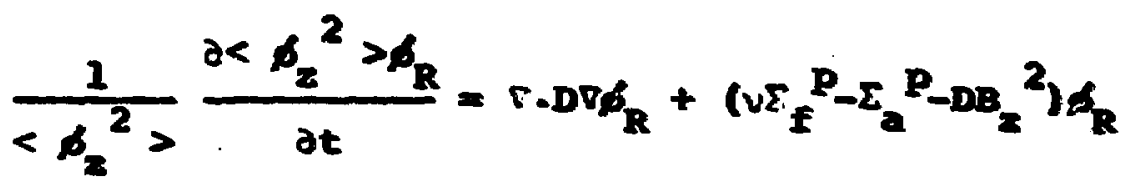


where

$$
v \Sigma_{E}^{P}=v \Sigma_{E}^{O}\left[1+\bar{a}_{1} T^{P}(x, t)+\bar{a}_{2}\left(\sqrt{T_{f}^{P}(x, t)+T_{0}}-\sqrt{T_{0}}\right)+\ldots\right]
$$

similarly for $\Sigma_{a}$ and $D$, and

$$
\frac{\bar{a}_{2}}{\bar{a}_{2}}=\frac{\left\langle\phi_{2}^{2}(2, t)\left(\sqrt{T_{f}(z, t)+T_{0}}-\sqrt{T_{0}}\right)\right\rangle}{\left\langle\phi^{2}(z, t)\right\rangle\left(\sqrt{T_{f}(z p(t))+T_{0}}-\sqrt{T_{0}}\right)} ;
$$

Eg. (5.15) defines a "special" axial D.W.F. relative to the axial peak fuel enthalpy. A similar "special" axial moderator WE appears in Eq. (5.13). How multiply both sides of Eq. (5.14) by $\phi\left(z_{p}(t)\right)$ and neglect the time variation of $b\left(z_{p}(t)\right) /<\phi_{z}{ }^{2}>$ to yield, droppirg the super " $p$ "

$\frac{\partial y}{\partial t}=\nabla \cdot D \nabla q+\left(w \Sigma_{E}-\Sigma_{a}-D B_{z}^{2}\right) Y$

This equation is - of course - precisely the usual separated form, but using the special weight factore for fuel moderator and coolant velocity, the calculated $y(x, t)$ is the radial power distzibution at tho shifting axial paak fuel enthalpy losation. 
Figure 7 shows the "special" axial D.W.F. defined by Eq. (5.15) for the 2.5\% to rod drop for two different delay times. The curves are very slowly varying up until very near the end of the transients and the flat value of 0.8 is undoubtedly an optimistic input to an $(r, t)$ calculation. Figure 8 shows the axial D.W.F. for the $3.8 \%$ rod arop for two different radial D.W.F.'s. These two tran-

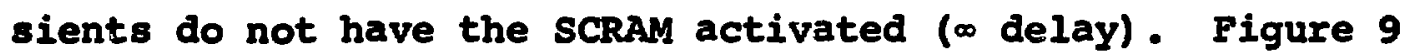
shows both the "regular" and "special" D.W.F.'s for an 0.27 second delay and a 2.5 second SCRAM time. The strong variation in the "regular" is clearly contrasted to the nearly flat behavior of the "special"; the single value of 0.85 is clearly optimistic.

Figure 10 shows the variation of the "velocity factor" during the $3.8 \%$ rod drop for the cases of $0 \%$ and $4 \%$ direct heat yield into the moderator. The structure of these curves is not simple and cannot easily be replaced by a constant, except that the value 1.4 clearly minimizes the moderator temperature rise and 0.4 (say) clearly maximizes it.

It is the near flatness of the "special" axial D.W.F. which recommends it for use in $(r, t)$ calculations, but the validity of such novel technique must rest on $(r, t)$ and $(r, z, t)$ comparisons. Such comparisons are fully discussed in Ref. 7.

In a case where moderator feedback is not accounted for, even though the temperatures are calculated, the velocity factor is worth only about $1 \mathrm{cal} / \mathrm{g}$ for the peak fuel enthalpy. 


\section{Validation of Separability}

Firstly, it is clear that a system with moving discrete rods is not spatially separable. The approximation $\phi(r, z, t)=$ $\phi(z, t) \Psi(r, t)$ cannot be strictly valid. Nonetheless, it is surprising how nearly separable such a systen really is in terms of the important parameters.

In order to secure an estimate of separability, the TWIGL code was extensively modified and subroutinized so that a moving central rod and separately moving SCRAM bank could be mocked up. The TWIGL two-group, 2D results were compared to the results obtained from the code NøAH which produced $(r, t)$ and $(z, t)$ calculations using only an average Ax.D.W.F. in the $(r, t)$ calculation and an average Rad. D.W.F. in the $(z, t)$ calculation.

\section{D.1 Eigenvalue Comparison}

The rod and SCRAM reactivity vs. time curve necessary* for the $(r, t)$ NøAF calculation was obtained by the selfweight method. 8 The $(r, t)$ and $(z, t)$ results were edited to produce a transient eigenvalue; in one group this is

*These functions $w_{i}(t)$ are not actually used but they are necessary to determine the $s_{i}(t)$ functions which are used in the $(r, t)$ calculation (see Ref. 7, pp. 9-17). 
$\lambda(t)=\frac{\left\langle\nu \Sigma_{f} \phi^{2}\right\rangle}{\left\langle\Sigma_{a} \phi^{2}+\overline{D B^{2}}{ }_{1} \phi^{2}+D(\nabla \phi)^{2}\right\rangle}-\Delta \lambda_{D O P}(t)$

where

$\Delta \lambda_{D O P}(t)=\frac{\left\langle\left[\phi^{2}\left(\nu \Sigma_{f}^{0} \alpha_{f}-\Sigma_{a}{ }^{0} \alpha_{a}-\overline{D B}{ }_{1}^{2}{ }_{1} \alpha_{D B}\right)-D^{0}(\nabla \phi)^{2} \alpha_{D}\right] f\left(T_{f}\right)\right\rangle}{\left\langle\left(\Sigma_{a}+\overline{D B^{2}}{ }_{1}\right) \phi^{2}+D(\nabla \phi)^{2}\right\rangle}$

the TWIGL results were similarly edited to produce a twogroup eigenvalue using an approximate two-group adjoint. This is calculated in the following way:

The time-dependent reactor equations for the flux $f(\underline{r}, t)$ in the usual two-group notation is

$D_{1} \nabla^{2} \phi_{1}-\Sigma \phi_{1} \phi_{1}+(1-\beta)\left(\nu \Sigma^{F}{ }_{1} \phi_{1}+\nu \Sigma_{2}^{F} \phi_{2}\right)+$ delayed neutron source $=$ $\frac{1}{v_{1}} \frac{\partial \phi_{1}}{\partial t}$

$D_{2} \nabla^{2} \phi_{2}-\Sigma_{2} \phi_{2}+\Sigma^{s}{ }_{1} \phi_{1}$

+ delayed neutron source $=$ $\frac{1}{v_{2}} \frac{\partial \phi_{2}}{\partial t}$ 
plus equations for delayed neutrons. The coefficients are time dependent because of feedback.

We define a time-dependent eigenvalue $\lambda(t)$ and the corresponding flux $Y(\underline{\underline{x}}, t)$ by means of equations (5.19):

$$
\begin{aligned}
& D_{1} \nabla^{2} \psi_{1}-\Sigma_{1} \Psi_{1}+\frac{1}{\lambda}\left(\nu \Sigma^{F}{ }_{1}{ }_{1}+\nu \Sigma^{F}{ }_{2} \psi_{2}\right)=0 \\
& D_{2} \nabla^{2} y_{2}-\Sigma_{2} y_{2}+\Sigma^{S}{ }_{1} \psi_{1}=0
\end{aligned}
$$

In equations (5.19) the coefficients are the same as those in the time-dependent equations (5.18). Equations (5.19) could be solved at each time point, but there is a simpler method of getting the eigenvalue.

We define an adjoint flux $\underline{y}^{*}(\underline{\underline{x}}, t)$ which is the solution of the equations adjoint to $(5.19)$. That is, $\psi^{*}$ satisfies equations $(5.20)$ :

$$
\begin{aligned}
& D_{1} \nabla^{2} \psi_{1}^{1 *}-\Sigma_{1}{ }_{1}{ }^{*}+\frac{1}{\lambda} \nu \Sigma^{F}{ }_{1} \psi_{1}{ }^{*}+\Sigma^{S}{ }_{1} \Psi_{2}{ }^{*}=0 \\
& D_{2} \nabla^{2} \psi_{2}{ }^{*}-\Sigma_{2} \psi_{2}{ }^{*}+\frac{1}{\lambda} \nu \Sigma^{F}{ }_{2} \psi_{1}{ }^{*}=0
\end{aligned}
$$

Then we take

$$
\begin{aligned}
\lambda= & \int \Psi_{1}{ }^{*}\left[\nu \Sigma^{F}{ }_{1} \Psi_{1}+\nu \Sigma_{2}^{F}{ }_{2}{ }_{2}\right] d \underline{x} / \int\left[\Psi_{1}{ }^{*}\left(-D_{1} \nabla^{2} \psi_{1}+\Sigma_{1} \Psi_{1}\right)+\right. \\
& \left.+\Psi_{2}^{*}\left(-D_{2} \nabla^{2} \Psi_{2}+\Sigma_{2} \Psi_{2}-\Sigma^{S}{ }_{1} \Psi_{1}\right)\right] d \underline{r}
\end{aligned}
$$


When $y$ is the correct solution to equations (5.19) or when $y^{*}$ is the correct solution to equations $(5.20)$, this expreseion gives the correct $\lambda$. When $y$ or $y^{*}$ differ from the correct solutions, the error in equation (5.21) is only of the second order in that difference. Bence, if we use fairly good estimates for " and $\psi^{*}$, expression (5.21) will give $\lambda$ with good accuracy. We get these entimates as followe.

We take $y_{1}=\phi_{1}$ and $y_{2}=\phi_{2}$, the time-dependent solution of equations $(5.18)$. we take $y_{1}^{*}=b_{1}$ and $y_{2}^{*}=c b_{2}$. Experience indicates that the spatial distribution of $y^{*}$ is given pretty well by this relation. We want to choose the constant $c$ to make the spectrum of the adjoint (i.e. $y_{2}{ }^{*} / Y_{1}{ }^{*}$ ) as good as poseible.

saking use of the form assumed above for $y^{*}$ and using the second equation from each pair of equations (5.19) and (5.20), we find

$$
c=\frac{1}{\lambda} \frac{\nu \Sigma^{B}{ }_{2}{ }_{1}}{\Sigma^{s}{ }_{1} \beta_{1}} \text {. }
$$

However, since this cannot be true at every space point, we try to satisfy it on the average by taking the mean values of the numerator and of the denominator. It is necessary to choose a weighting function to obtain these mean values; we have chosen $\phi_{2}$ as the weighting function. Other choices are possible, of course, but we have not investigated them. 
Then we have

$$
c=\frac{1}{\lambda} \frac{\int v^{\mathrm{F}} \phi_{2} \phi_{2} d x}{\int \Sigma^{s} \phi_{1} \phi_{2} d x}
$$

Then

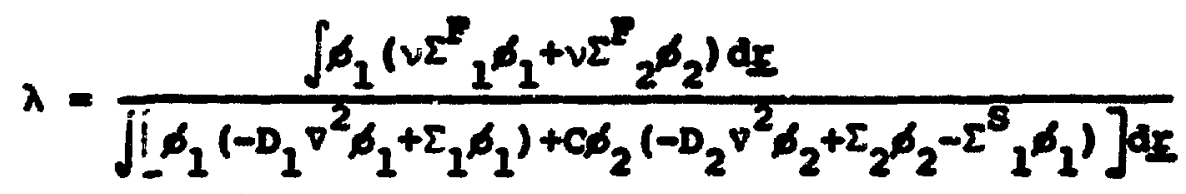

By differentiation of this expression under the integral sign, we can find the separate contributions to the reactivity from various mechaniems. For example, tha Doppler effect, due to crose-section changes produced by fuel temperature alterations is - after some algebra -

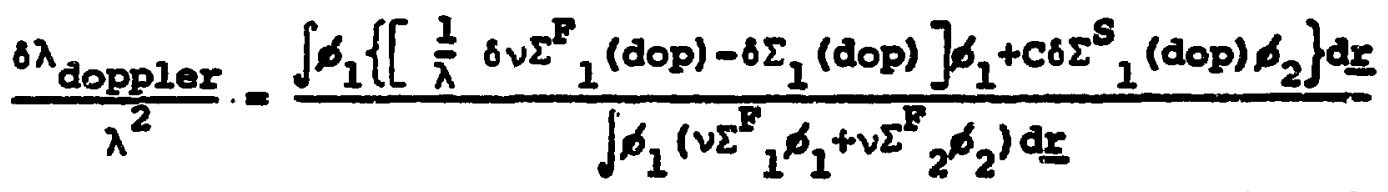

By similar means, one can separate the rod inserted positive reactivity from the SCRAM bank negative reactivity. We only compare.their sum. This is done in Fig. 11. It can be seen 
that the is calculations exhibit elightly different edite for $(\lambda-1) / \lambda$. It is clear that the feedback eflects in the $(x, t)$ calculation tend to decrease the feedback worth of the dropped rod (this has been discusend in som dotall in Ref. 7) but it is not completely clear why no (or nearly no) such effects appar in the 2D, two-group Iwsel adit which has very nearly the sam time behavior as the 10, one-group $(z, t)$ calculation. Nonetheless, the amil differences (< $10 \%$ in $\Delta \lambda / \lambda$ at the peak) show that the reactivity effect of the rod and bank are spatially separable.

\section{D.2 Bower Poak/Average Comparison}

The IWICL calculated power $P(x, z, t)$ can be averaged radially or oxially to yield $\bar{p}(z, t)$ and $\bar{p}(x, t)$, erom these, the average radial and axial peak/average pownes can be campared to those calculated by whis. Inis is done in Fig. 12. The results here are also quite good and show about the sam $10 \%$ difference between the Isph resulte and the averaged TwIel calculations.

\section{D.3 Doppler W.F. Compurison}

The $\Delta \lambda_{D O P}(t)$ as calculated by TwIGE, and indeed nonlinear feedbeck in goneral, is not esparable except under very stringent circumstances. In particular, if $\phi(x, z, t)=$ $\phi(x, t) \phi(z, t)$ and one can assume $T_{f}(x, z, t)=\Delta T(x, t) \Delta T(z, t)+$ $\mathbf{T}$, then 


$$
\left\langle\rho^{2}\left(\sqrt{A_{T}(x, t) A^{2}(x, t)+m_{m}}-\sqrt{T_{m}}\right)\right\rangle
$$

is certainly not accurately aparable for $\mathrm{I}_{\mathrm{f}}-\mathrm{I}_{\mathrm{II}}>3 \mathrm{I}_{\mathrm{I}}$ indeed, because of this we expect that the comparisen of the wirel D.W.F. and the product of the N,Ma Ax.D.W.F. and Rad.D.W.F. should be a poor one, nepecially for long time when $I_{f}$ is large. In Fig. 13 we mike this comparison. The discrepuncy for mal1 tines is about $15 \%$, at 0.8 seconde the exror reaches 30\%, but by the time peak powne has been reached and for the rest of the relevent calculation the exror remains at from 10 to $12 x$.

In order to explain - or at least offor a poseible explanation for this result - we have to invoke the consapt of local seperability. wile it is true that the whole fluxes, temperatures, etc. are not separable, it seens clear from perspective viows of $P(x, a, t)$ that only a amall portion of the core near the central control rod has a high inportance. This may mean that in this small portion of the core the fluxes, temperatures, etc. are indend separable. This point is discusead in nore detail in Ref. 7. 
vI Cancluetions:

The determination of a W.F. by Flux-adjoint veighting of the feedback function (Bq. (1.4)) producas a correct separation of spatial dimension st leat for slow transiente. Phis is clearly dumonstrated by the explanation of the paradoxical pearce 1 and lese ${ }^{2}$ results. such a W.F. can bo calculated during a transient by means of Eg. (1.4) which yielde an instantaneous statistical W.F. .

The degree of acsuracy obtained from id calculatione, even with average transverse W.F." in a highly nonseparable rod drop accident followed by sensh, leads us to expect that fewer than 3D calculations can be ganerally expected to lead to very nearly the sane resulte that a full 30 model would lead to. Inis is demonstrated quite clearly in mef. 7. 


\section{References}

1. R. H. Pearce, Xenon Oscillations in Finite Reactors, kucl. Sei. Eng. 16, 336 (1963)."

2. R. R. Iee, Maltidinensional Effects in the Analysis of Xenon Spatial stability, Jrans. Amex. Mucl. Soc. 12, 2 (1969).

3. R. E. Ginna, Buclear Pover plant bait Bo. 1, Final Facility Deseription and Safety daelyais Report, Vol. 3 $14,2.6-7,8$.

4. D. I. Wast et al., bse of the Malibatic Apprexination in Prompt-critical, Harge core mactor bxeuraion palyses, Irans. Nmex. Muel. Soc. 2, bo. 2, 497-498 (1965).

5. J. E. Wood, Mnalyais methods of bpothetical supex-

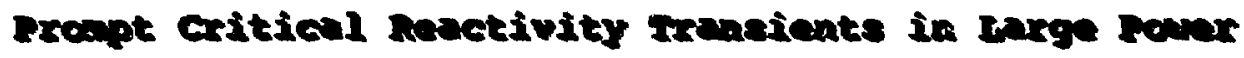

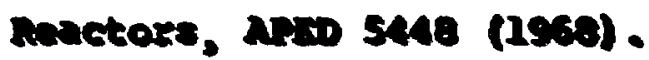

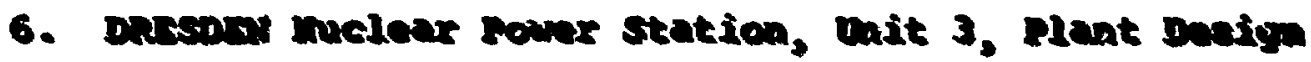
and analyeis Report, Rnadinat no. 3.

7. 6. S. Iellovehe, at al., Rod prop and semn in wouling inter maetors, R-1021 (in preparation).

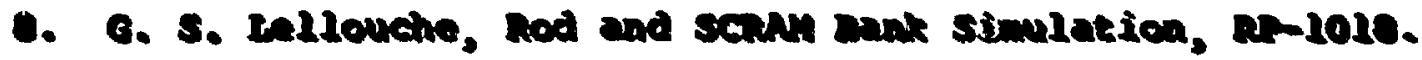




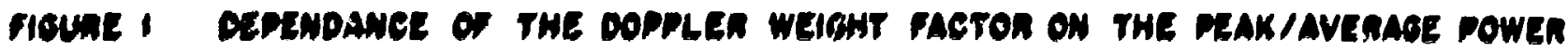

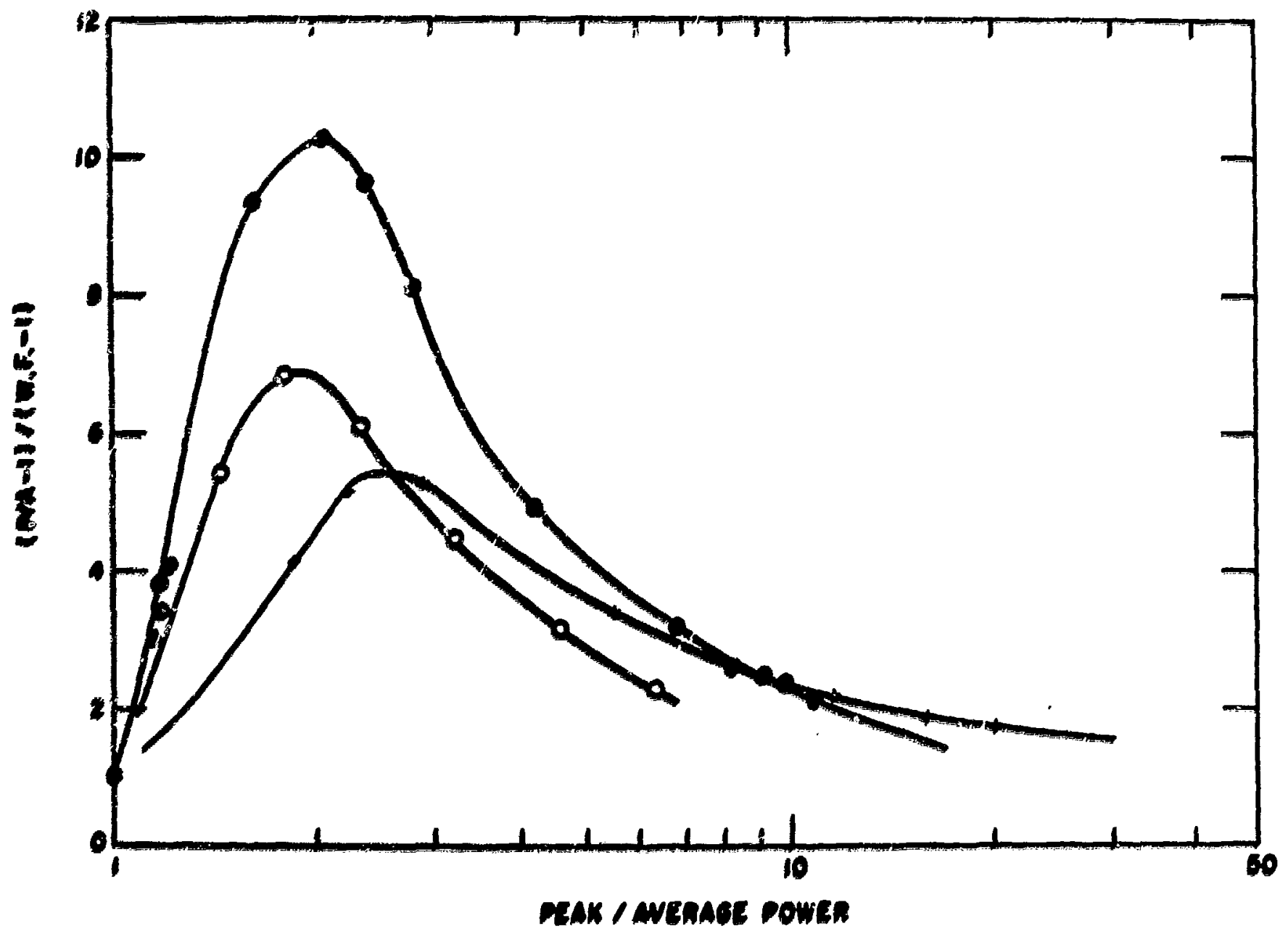


FIGURE 2 THE EFFECT OF AN UNRODDED ANNULAR REGION ON CENTRAL ROD WORTH

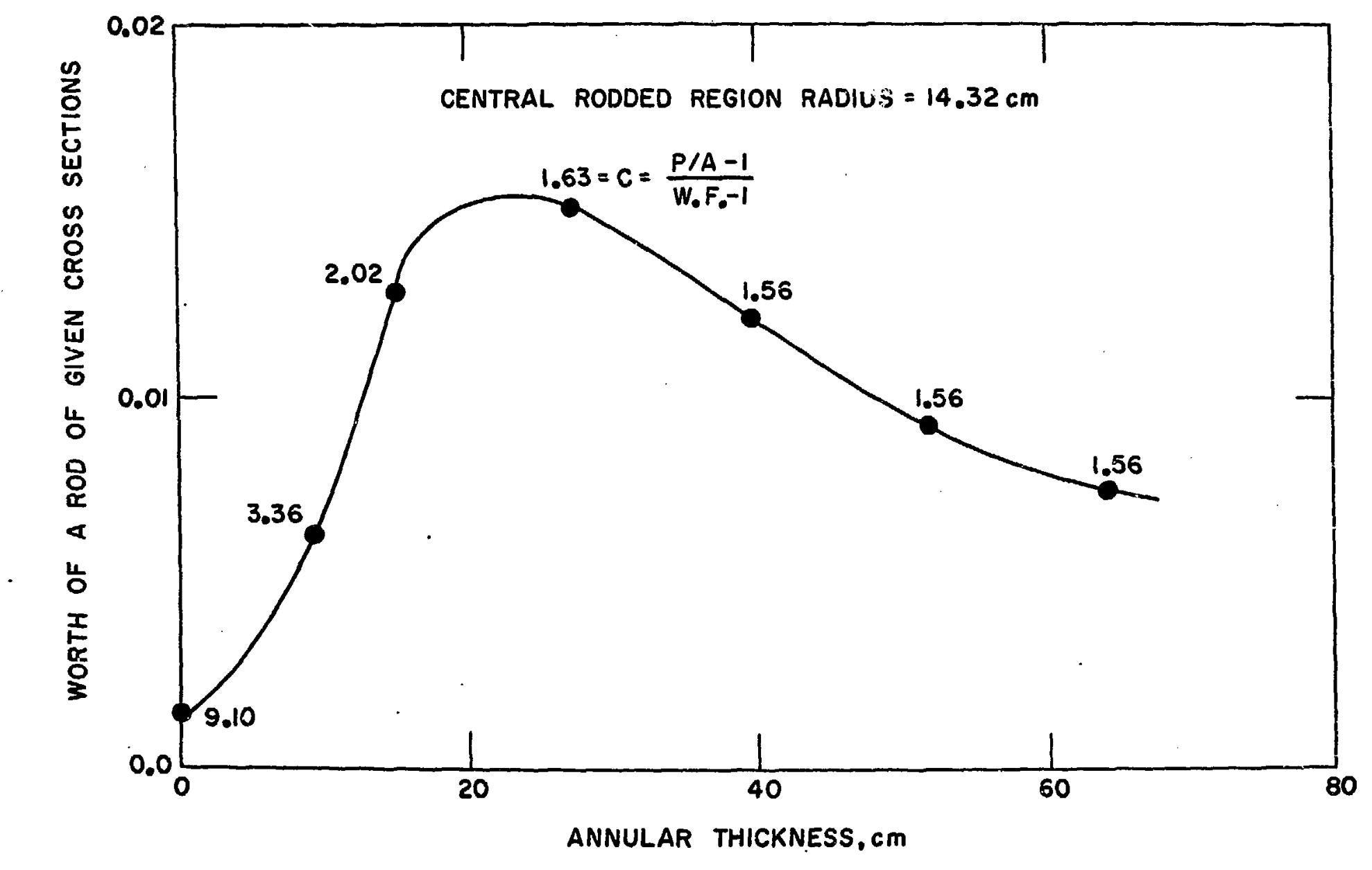


FIGURE 3 SOME TRANSIENT CHARACTERISTICS OF THE $2.5 \%$ ROD DROP ACCIDENT IN A B.W.R.

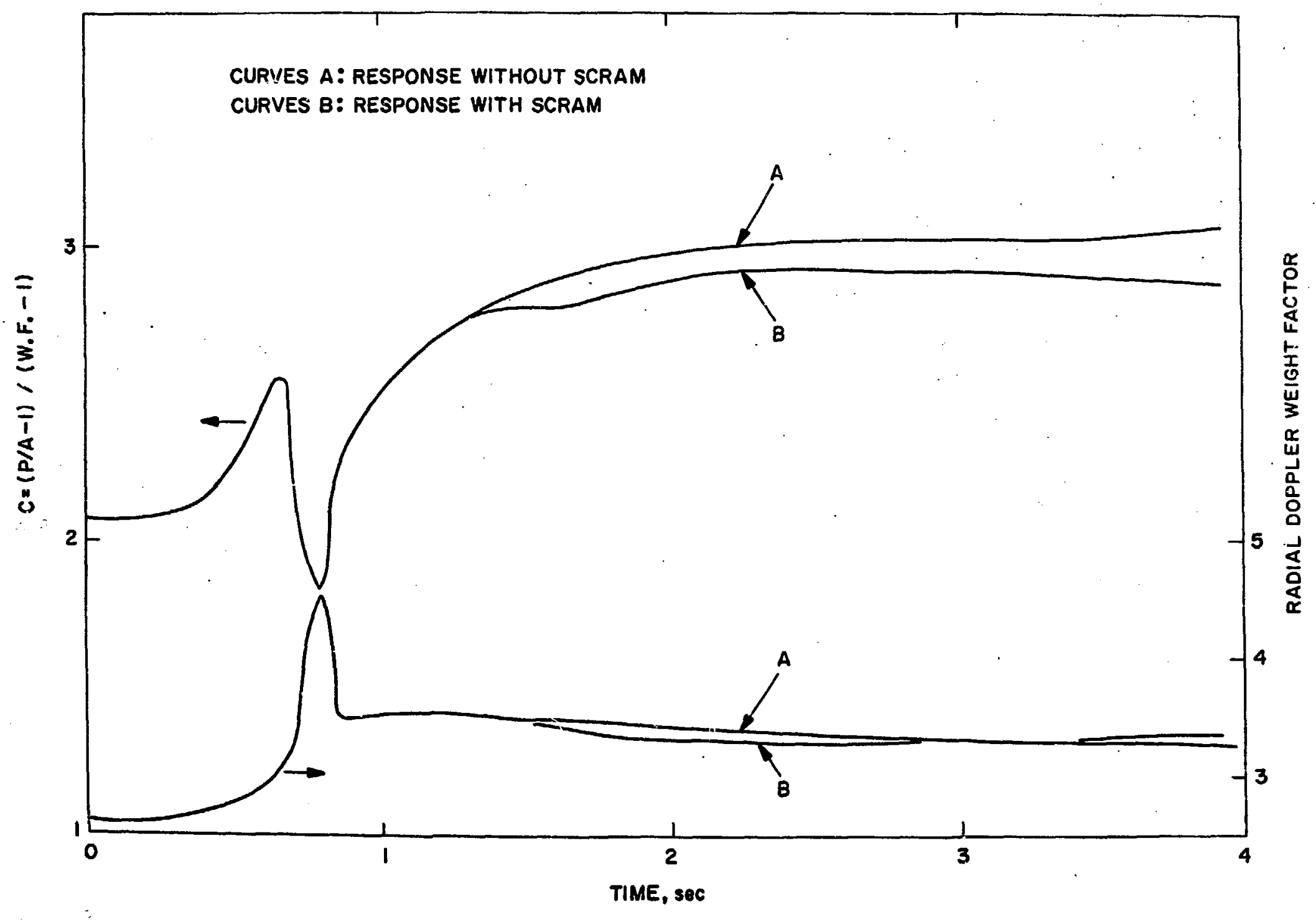


FIGURE 4 SOME TRANSIENT CHARACTERISIICS OF A ROD EJECTION FROM FULL POWER IN A PWR

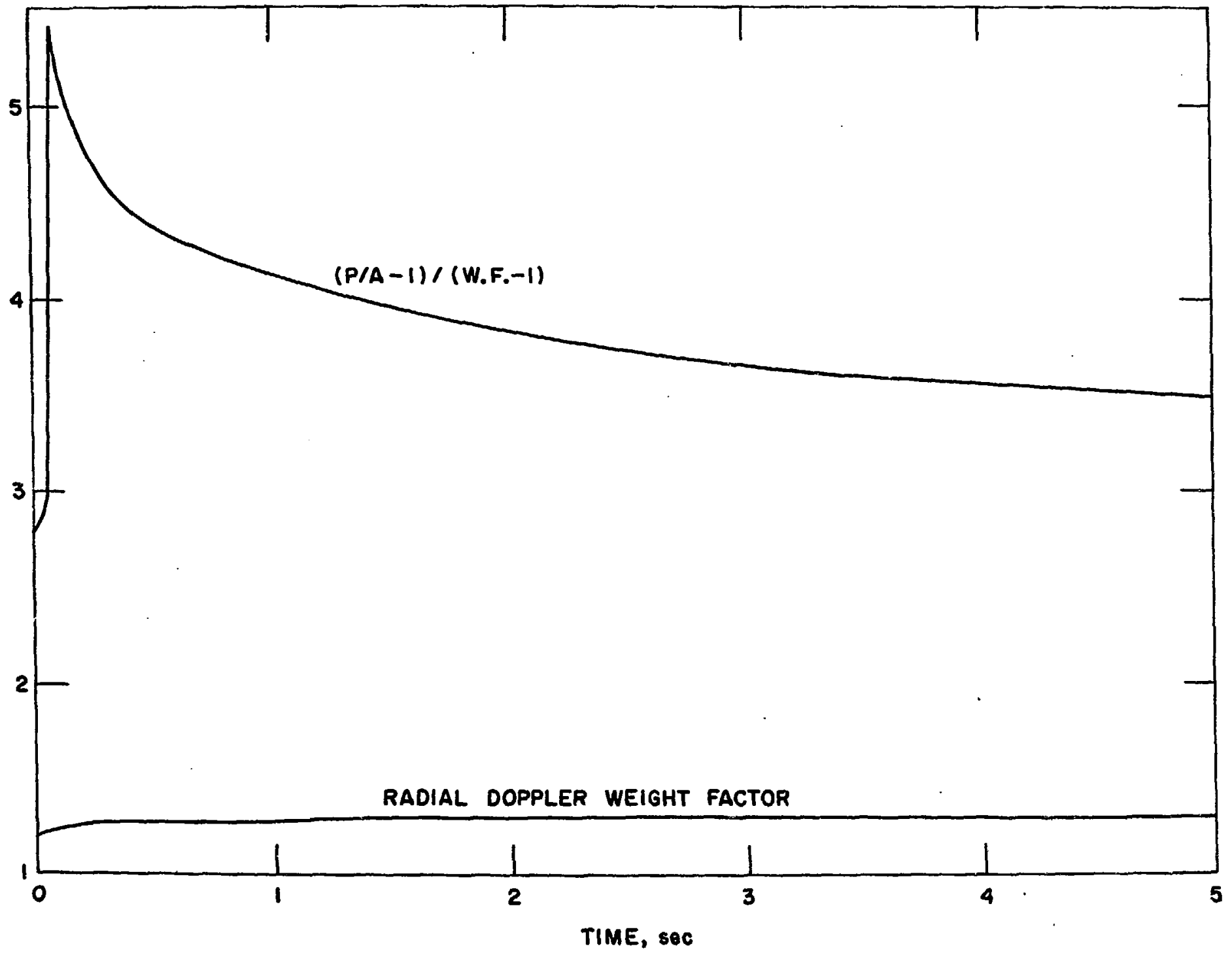


FIGURE 5 VARIATION OF THE AXIAL DOPPLER WEIGHT FAGTOR DURING THE $2,5 \%$ ROD DROP IN A B,W.R.

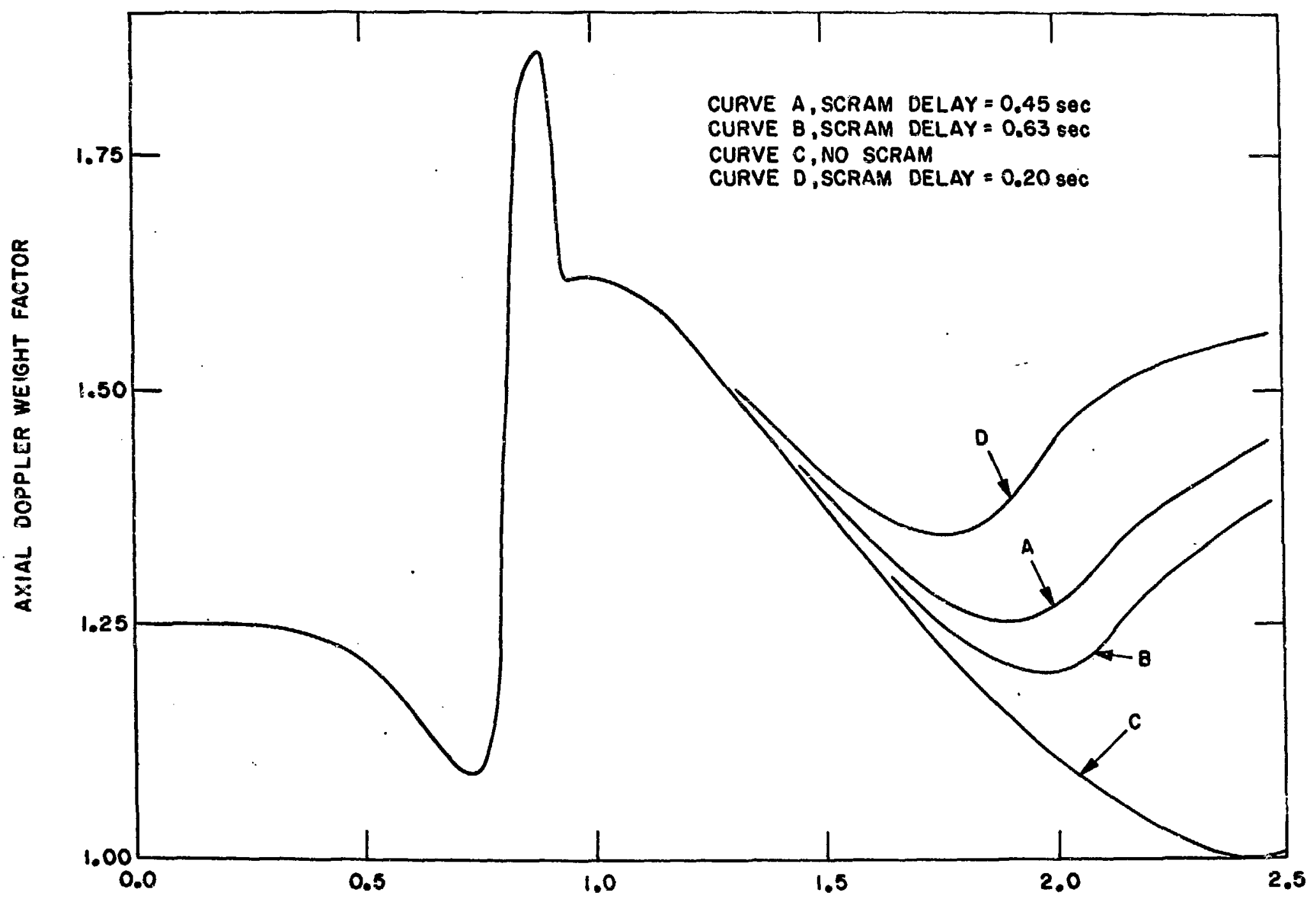

TIME INTO THE TRANSIENT, seC 
FIGURE 6 TRANSIENT AXIAL DOPPLER WEIGHT FACTOR AS A FUNCTION OF AVERAGE FUEL TEMPERATURE RISE FOR THE $2.5 \%$ B.W.R. ROD DROP ACCIDENT

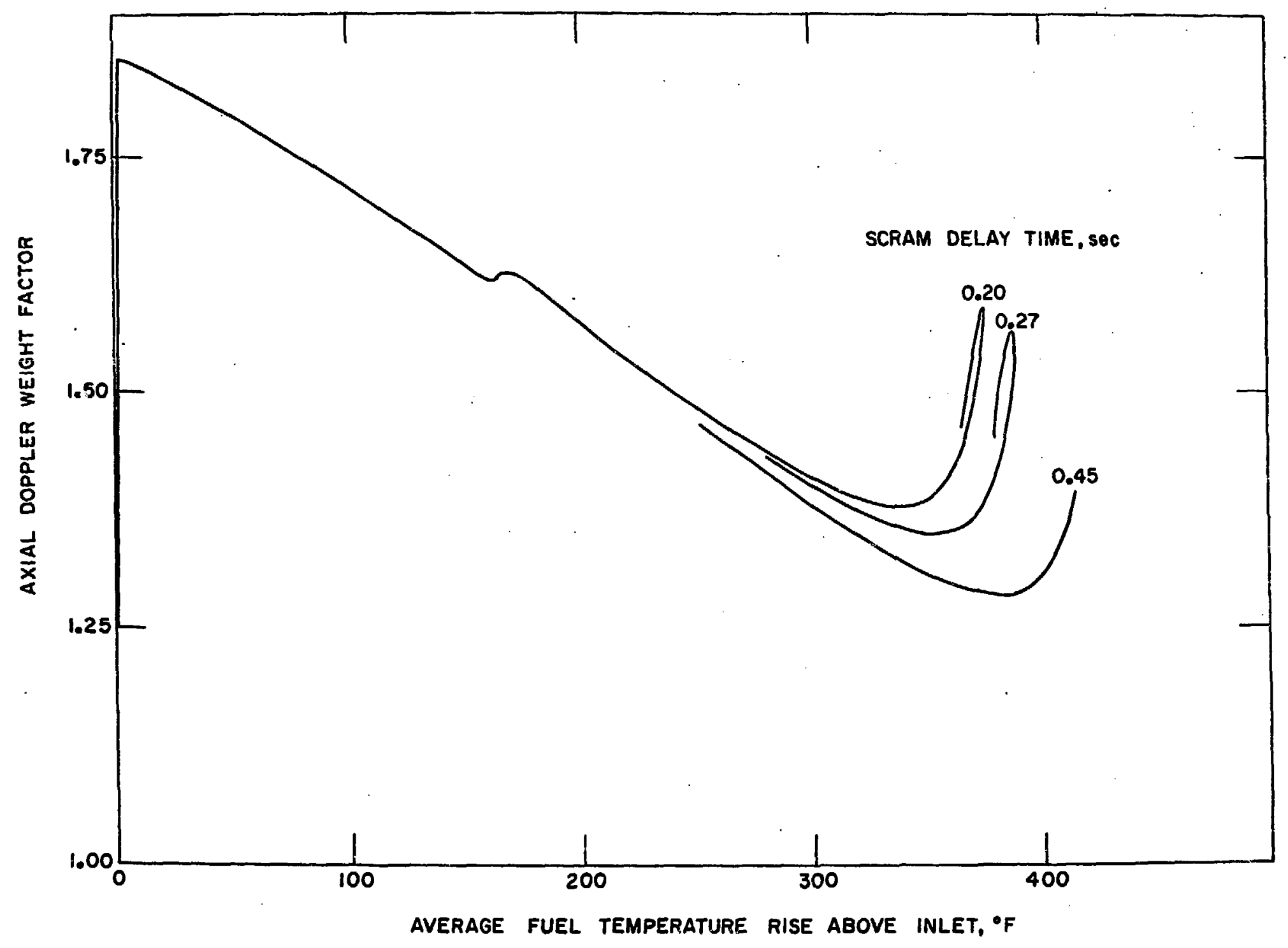


FIGURE 7 AXIAL DOPPLER WEIGHT FACTOR FOR USE IN HOT SPOT CALCULATIONS (SEE TEXT FOR DEFINITION) DURING THE $2.5 \%$ ROD DROP

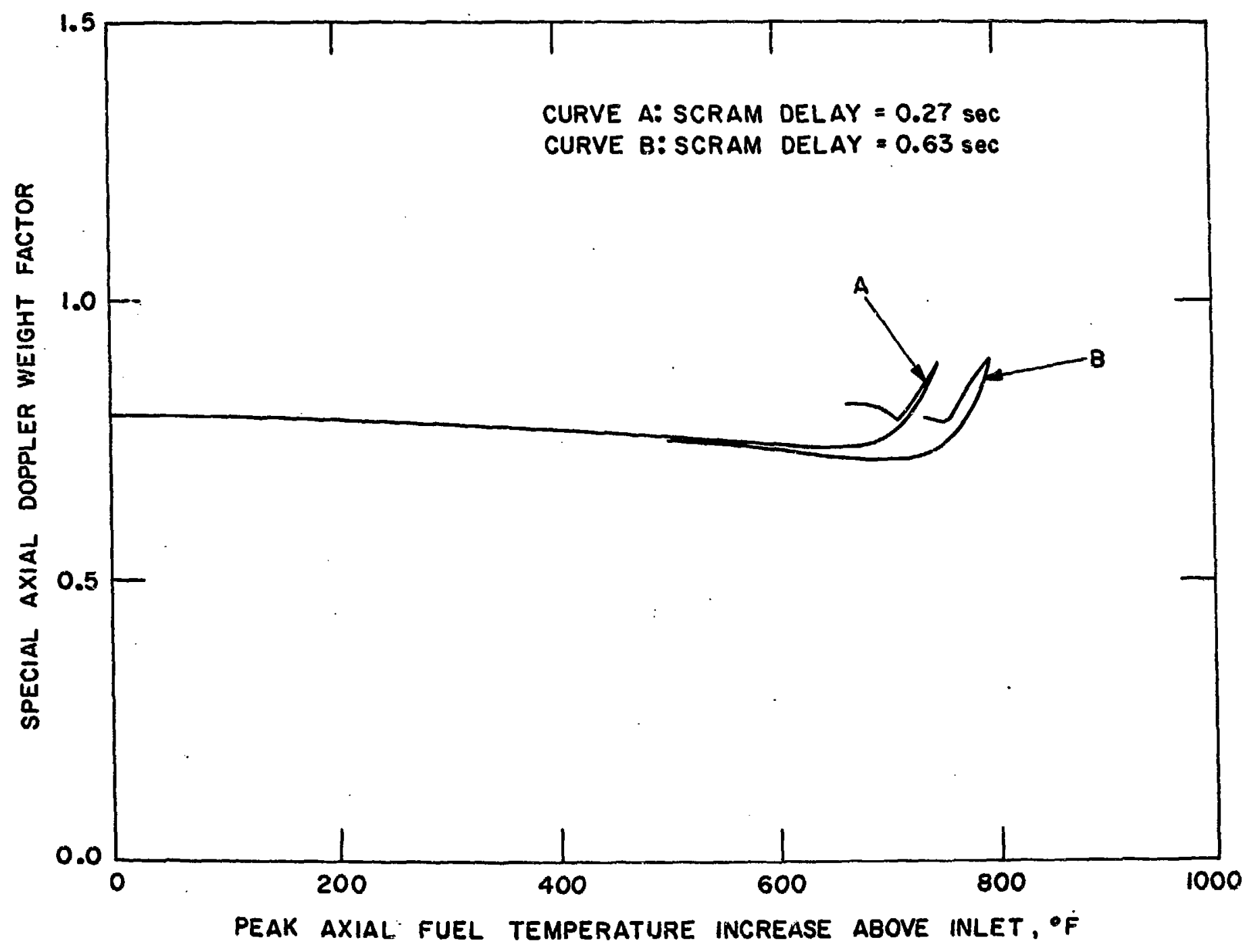


SPECIAL AXIAL WEIGHT FACTOR

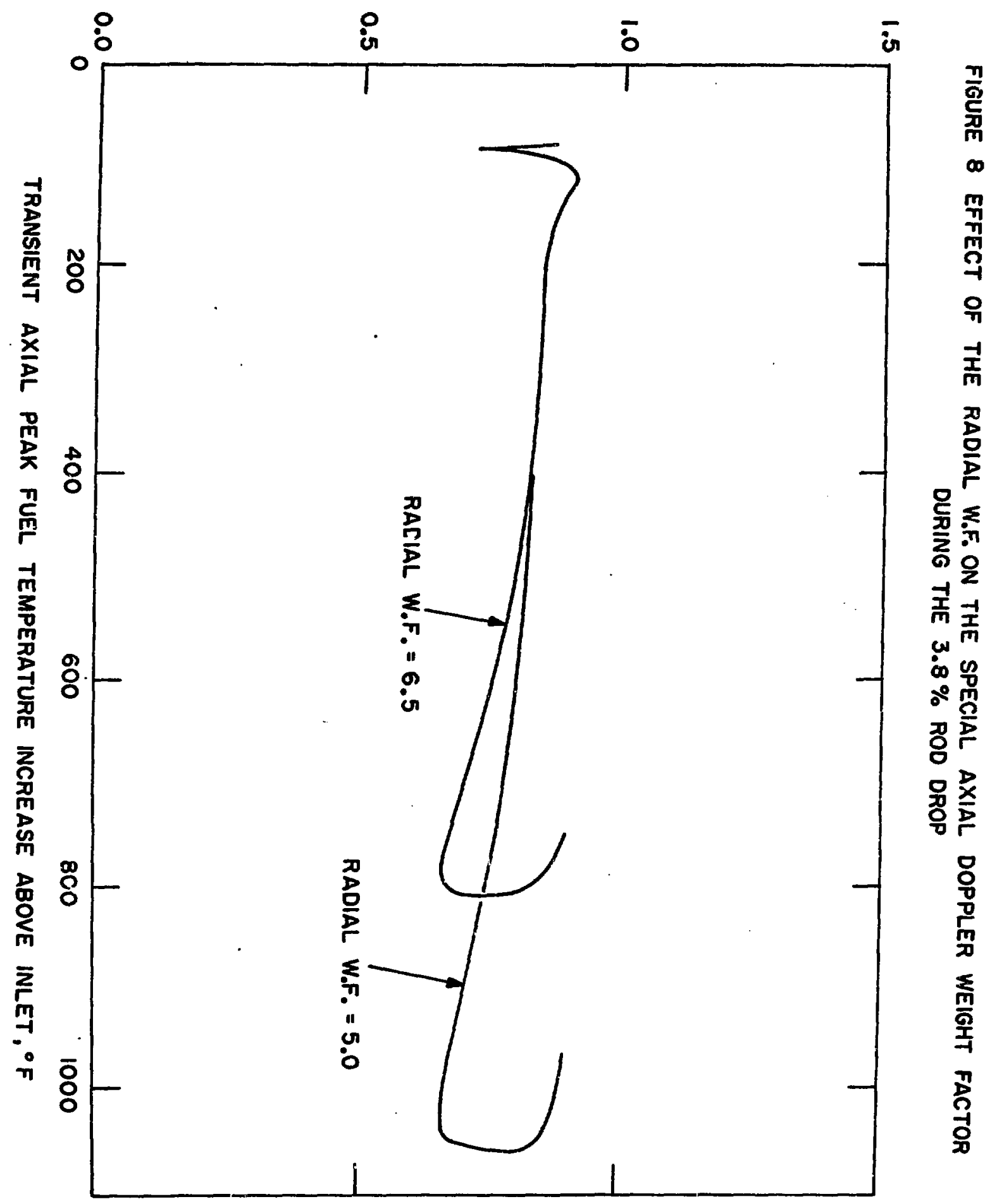


FIGURE 9 VARIOUS WEIGHT FACTORS FOR THE $3.8 \%$ ROD DROP: 0.27 seC DELAY, 2.5 SOC SCRAM, SELF WEIGHT . RADIAL DOPPLER WEIGHT FACTOR $=5.0$

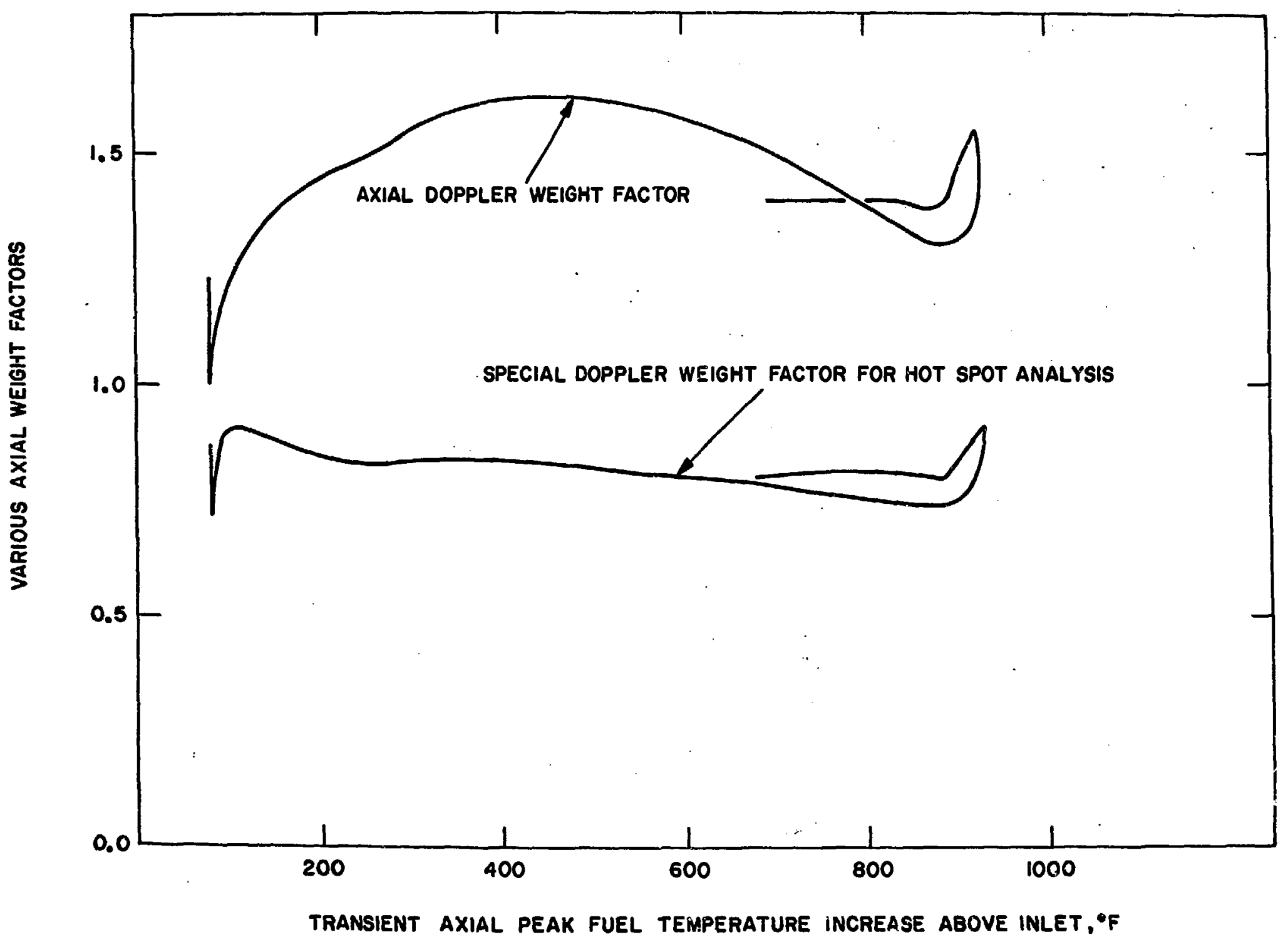


FIGURE IO MODERATOR VELOCITY FACTOR FOR USE IN DIRECT HOT SPOT CALCULATION

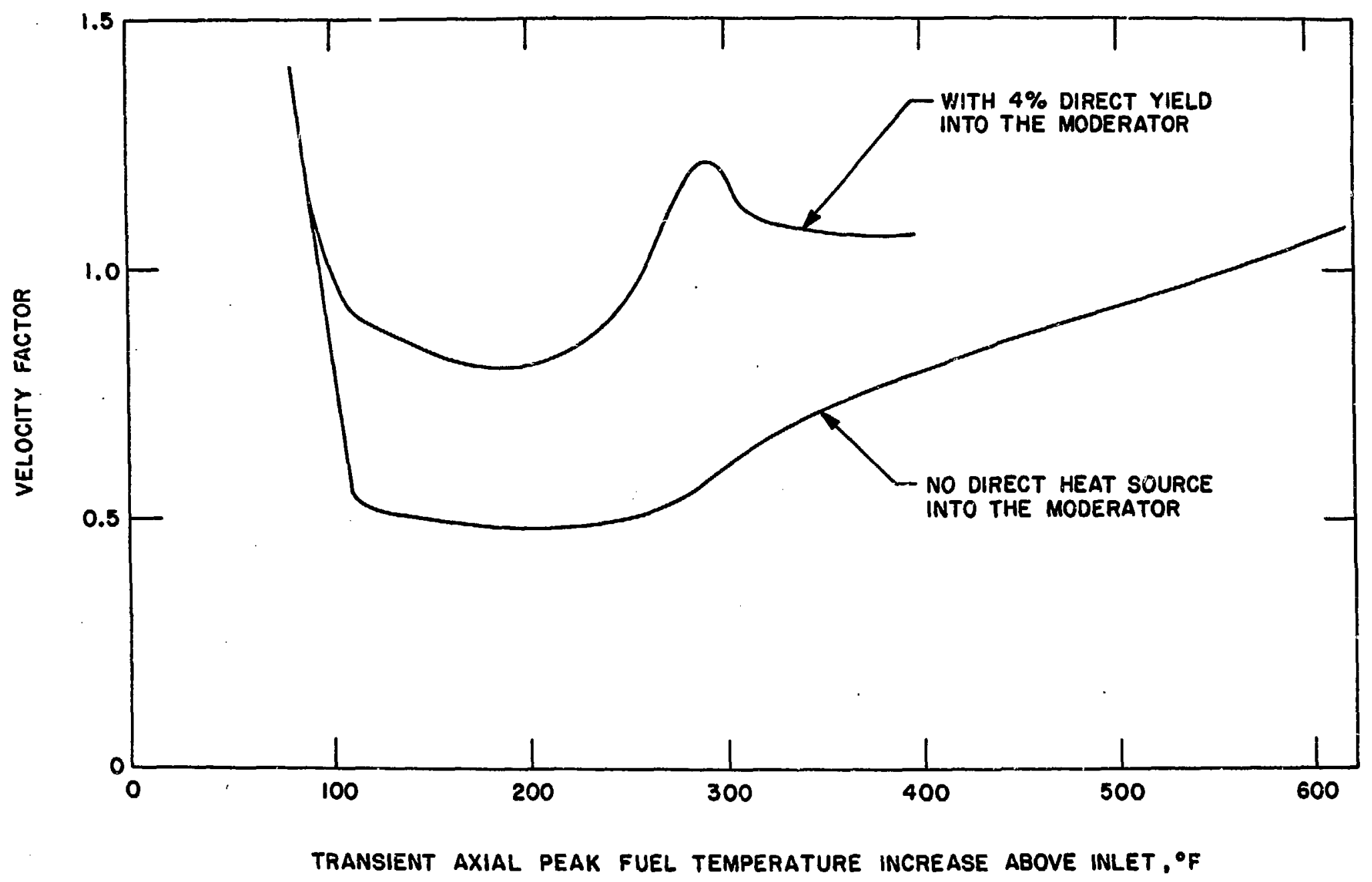


FIGURE II ROD-SCRAM BANK INTERACTION, RESULYYS FROM NDAH AND TWIGL INCLUDING FEEDBACK $2.5 \%$ ROD DROP ACCIDENT

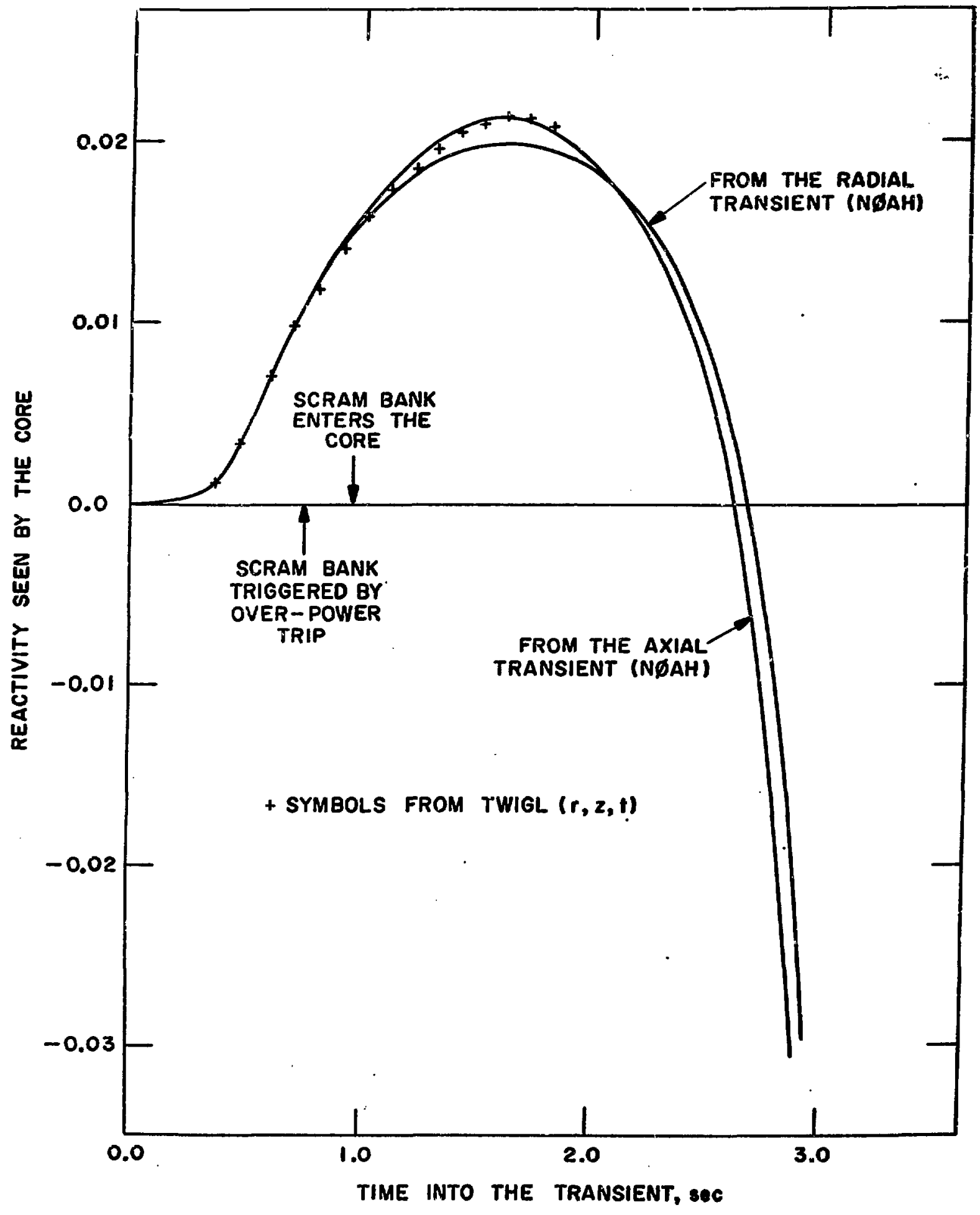


aXIAL PEAK / AVERAGE POWER

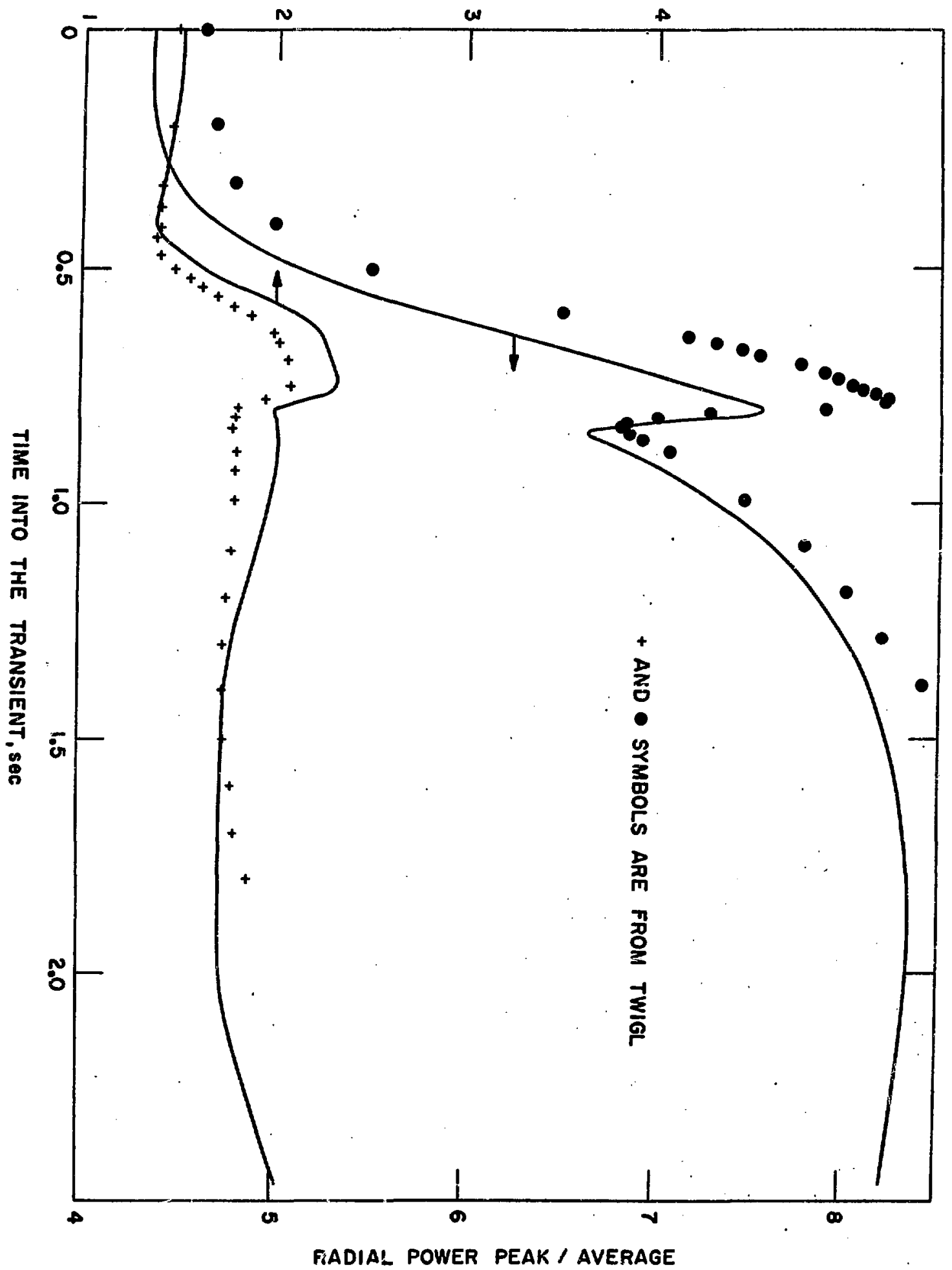

$\frac{\pi}{2}$
$\frac{2}{0}$
n

옹 일

另몽

ㅇํำ

b क

2 을

可

m

2

2 ำ

온

0

学

m

$c=5$

얼롱

공

개욤ㅇ

类舟

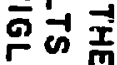

○요

$5 \frac{1}{8}$

을

$5 \pi$

20

을 꿍

잊

3

콯

m

m

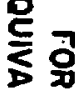

$\sum_{j}$

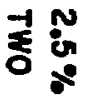

FADIAL POWER PEAK / AVERAGE 
FIGURE 13 A COMPARISON OF THE TWIGL W. F. AND THE PRODUCT OF THE NDAH W.F.

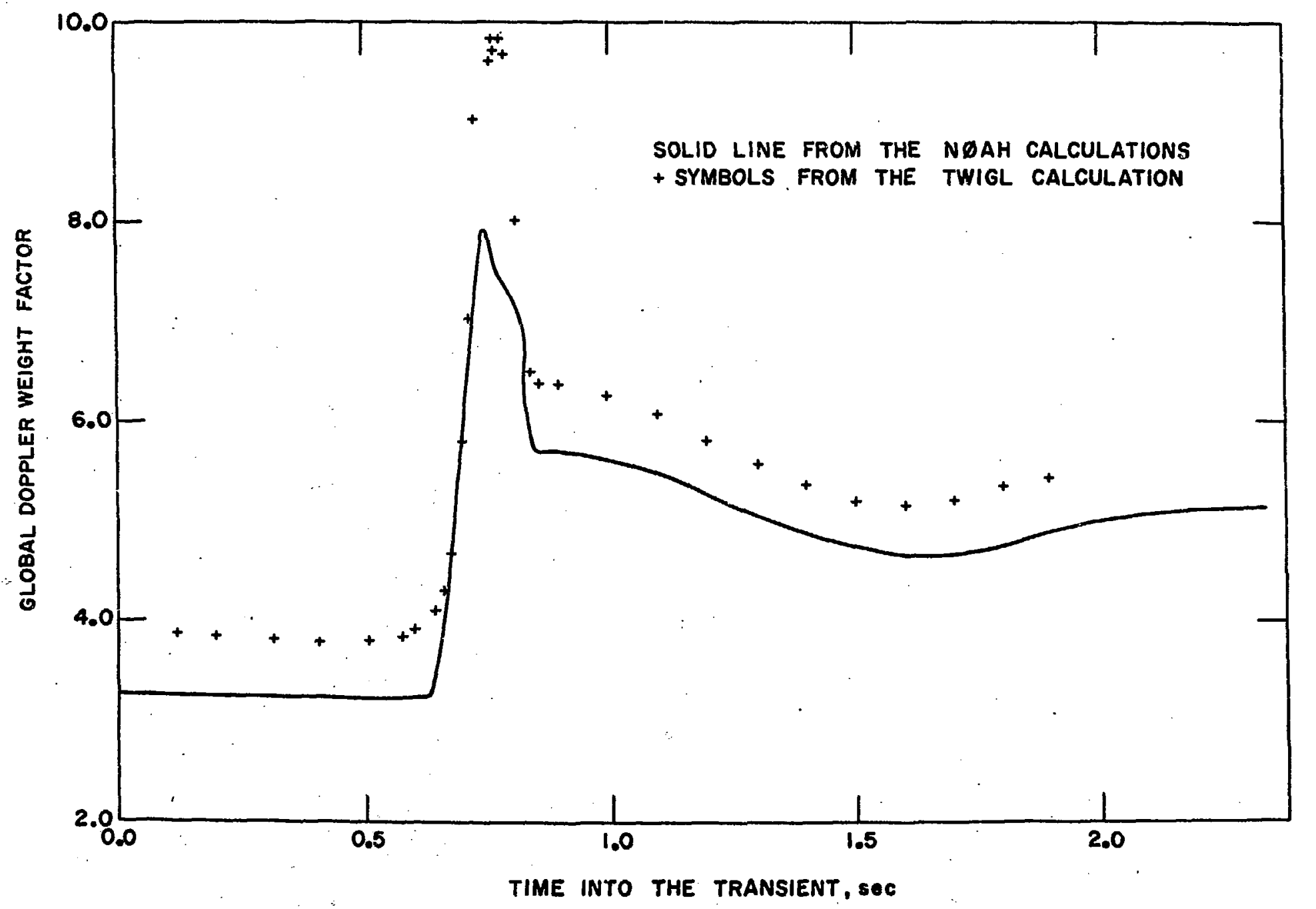

